\title{
LARGE DEVIATIONS FOR TANDEM QUEUEING SYSTEMS ${ }^{1}$
}

\author{
ROLAND L. DOBRUSHIN \\ and \\ EUGENE A. PECHERSKY ${ }^{2}$ \\ Institute for Problems of Information Transmission \\ Russian Academy of Sciences, 19 Ermolovoj Str. \\ GSP-4 Moscow, 101447 RUSSIA
}

(Received May, 1994; revised July, 1994)

\begin{abstract}
The crude asymptotics of the large delay probability in a tandem queueing system is considered. The main result states that one of the two channels in the tandem system defines the crude asymptotics. The constant that determines the crude asymptotics is given. The results obtained are based on the large deviation principle for random processes with independent increments on an infinite interval recently established by the authors.
\end{abstract}

Key words: Delay, Large Deviations Principle, Processes with Independent Increments, Networks, Tandem Queueing System.

AMS (MOS) subject classifications: 90B15, 60F22.

\section{Introduction}

Within the framework of the queueing theory, deep analytical methods have been developed for investigating statistical characteristics of waiting times for messages under several disciplines. However, these methods turned out to be of little use in the area of communication networks, where a message is served in a consecutive order by several devices. The simplest example of this situation is a tandem consisting of two consecutive channels (or devices) with the simplest service discipline FIFO (first in = first out). Here very cumbersome explicit solutions were found for some special cases only. In [4], an explicit solution was found for the case of a Poisson input flow with service time exponentially distributed. In this case, the output flow from the first device appears to be again Poisson. Explicit solutions were also found for the cases when service times are deterministic (see $[10,12])$ and when the service times for both servers are identical $([2,3])$. There are no analytical results for the general situation.

More restricted formulations of the problem can be of practical interest. For example, in many real situations, the designer of the system sets a significance level, i.e., a small number $p$, and wants to make sure that the waiting time $\omega$ of a message in the system exceeds a given bound with probability smaller than this significance level $p$. It means that it is of interest to study the function $T(p)$ defined by the relation

\footnotetext{
${ }^{1}$ The work is partly supported by the Russian Foundation of Fundamental Researchers,
} Grant 93-011-1470.

${ }^{2}$ The work of the second author is supported in part by a grant of AMS-FSU Aid Fund. 


$$
\operatorname{Pr}(\omega>T(p))=p
$$

in the stationary mode for a servicing system.

If we suppose that the decrease rate of the tail of the probability distribution describing the input flow is exponential or quicker, it is reasonable to expect the following asymptotics of the function $T(p)$ :

$$
\lim _{p \rightarrow 0} \frac{T(p)}{\ln (1 / p)}=\alpha
$$

So $T(p) \approx \alpha \ln (1 / p)$ for small $p$, and it is of interest to find the constant $\alpha$. Of course, the following question arises: Is this approximation sufficiently accurate for applications? Here it is appropriate to emphasize that in the vast majority of cases only very rough estimates for the desired significance level can be extracted from the real situations. It is common to apply a very rough scale of the type

$$
p=10^{-2}, 10^{-3}, 10^{-4}, \ldots
$$

and so on. It means that only $\ln (1 / p)$ is given so that inaccuracy of the asymptotics of $T(p)$ is lost, when looking for inaccuracy of the significance level $p$.

Instead of the function $T(p)$ it is possible to study the inverse function $p(T)$. It is easy to see that

$$
\frac{1}{\alpha}=-\lim _{x \rightarrow \infty} \frac{\ln \operatorname{Pr}(\omega>x)}{x} .
$$

So the stated problem belongs to the class of problems involving exponential asymptotics of large deviations intensively studied in recent years (see the monographs [7] and [8]). In the framework of the theory of large deviations, $\alpha^{-1}$ is the minimal value of the rate function in the corresponding domain.

In the following, we shall assume that both the devices of the tandem obey the FIFO discipline. It means that each device begins processing a message either at the moment when the message arrives at the device, if it was idle or at the moment when the service of the previous message is complete. In the monograph [1], such service disciplines were called disciplines of type I. It was assumed that the moment of an arriving message at the second device coincides with the moment of completion of its service by the first device. Consider the random variable $\omega$ that describes the waiting time of a message, which we interpret as the virtual waiting time of a virtual message. (See, for example, [1].) This means that a virtual message requests the service times on both of the devices to be equal to zero and it is processed after all "real" messages entered the system earlier or the same time. We shall return to this notion in $§ 3$. The complete time that a real message spends in the system is a sum of the virtual time and the times that are necessary to process this message by both channels. A study of this complete time can be reduced to a study of the virtual time in an evident way and we restrict ourselves to a study of the virtual waiting time.

The main result of the paper states that if $\omega$ is the virtual delay of the message and the constant $\alpha$ is defined by relation (1.3) then

$$
\alpha=\max \left\{\alpha_{1}, \alpha_{2}\right\}
$$

where $\alpha_{1}$ and $\alpha_{2}$ give the solutions of the similar problems in which each device serves separately the original Poisson flow of the messages. A way of determining the constants $\alpha_{i}$ goes back to the Cramér papers [5,6] and it can be found in Borovkov [1]. In this monograph, explicit 
formulas for the characteristic function of the virtual waiting time, known for the case of a single device, are used for this aim. An alternative derivation of this result is explained in [9]. This alternative derivation is based on the general theory of large deviations.

Because $\omega=\omega_{1}+\omega_{2}$, where $\omega_{1}, \omega_{2}$ are the waiting times for the first and the second device, respectively, the result (1.4) may appear almost evident from a first glance. But it turns more surprising if we recollect that $\alpha_{2}$ was calculated under the hypothesis that the input flow to the second device is Poisson; this hypothesis is not valid for the intermediate flow in the tandem. In general, the constants $\alpha_{i}$ depend essentially on the statistics of the input flow. It is possible to oppose these arguments with an intuitive explanation. If the second device is "worse" than the first one, the fluctuations of the input process, critical for the second device, go without essential disturbances through the first device.

The derivation of the formulated result is based on the use of a quickly developing branch of the modern probability theory: the theory of large deviations. The main theorems of this theory state that, for wide classes of random processes and sets of their realizations, the logarithms of the probabilities of these sets are asymptotically defined by minima of a special function, which is called the rate function, over these sets. However, a direct application of such results to the processes arising in queueing theory seems to be difficult. These processes are complex, so it is hard to find explicit formulas for the corresponding rate functions, and it is even more difficult to find them for minima of the rate functions. Here we develop a roundabout approach. We explain it in the context of our tandem problem; it can also be applied to other problems in queueing theory.

Without loss of generality, we assume that the service times of messages for both devices are defined at the moments of their arrival to the system. So, if the input flow is Poisson, a twodimensional generalized Poisson process, with independent increments, arises as a description of the input to the system. In such an approach, the random variable $\omega$ turns out to be a functional of this input process. But simple explicit formulas for the rate functions of processes with independent increments are well-known. The domain in which we have to minimize the rate function becomes more complex. But it turns out that it is easier to minimize a simpler function over a more complex domain than to minimize a more complex function over a simpler domain.

Though the large deviations for processes with independent increments have been studied intensively enough, it turns out to be impossible to apply the known results to the considered case. This is why in [9] we propose a new version of the theorem on large deviations suitable for the considered case. Here we only formulate and apply this theorem. We cannot use the known theorems for several reasons. Most pertinent papers (see $[15,16])$ are devoted to the study of large deviations for processes with independent increments defined on a finite time interval but in the typical problems arising in our approach, it is necessary to study the functionals depending on the behavior of the process on an infinite interval. There are some papers $[13,17]$ in which theorems on large deviations on the infinite intervals are formulated with the use of some topologies defined by projective limit constructions, but such topologies are too weak for our goals (see the discussion in $\S 7$ of [9]). Another difficulty is due to the assumption used by many authors that the moment generating function of the increments of the process exists for all values of the argument of the function. It excludes the most popular case of an exponential distribution for service time from consideration. And finally, we need a theorem on large deviations for the vector-valued processes.

We shall not be concerned here with the problem of studying queueing length in a tandem. A result for the large deviations of the queueing length is formulated in Walrand's monograph [20] without a rigorous proof (more precisely, Walrand considered values of a total population in the tandem system). The large deviations principle proved in [9] seems to be applicable also for such functionals. The paper of Tsoucas [19] is devoted to a proof of this result for the special case of 
exponential service times; however, the author did not take into consideration one of the difficulties mentioned above, namely that the random functionals under consideration are defined on an infinite interval. (Also, the author applied for the proof, a sequence of functionals on the Skorokhod space and treated them as continuous. However, these functionals can be discontinuous at some point of a Skorokhod space.)

\section{The main result}

We describe the input flow of a tandem as a sequence of independent three-dimensional random vectors

$$
\Xi=\left(\tau^{i}, \xi_{1}^{i}, \xi_{2}^{i}\right)_{i=-\infty}^{\infty}
$$

with nonnegative components. We interpret the random variables $\xi_{1}^{i}$ and $\xi_{2}^{i}$ as the service times of the $i$ th message at the first and second device, respectively, and $\tau^{i}$ as the $i$ th interarrival time.

Make the following assumptions.

(1) The sequence $\Xi$ consists of identically distributed independent vectors. We suppose that

$$
\mu_{j}=\mathbf{E} \xi_{j}^{i}<\infty, \quad j=1,2
$$

(2) For every $i$, the three random variables $\tau^{i}, \xi_{1}^{i}, \xi_{2}^{i}$ are mutually independent.

(3) The random variables $\tau^{i}$ are exponentially distributed with parameter $\lambda>0$ (i.e., they have the probability density $\lambda e^{-\lambda x}$ ). (The last condition means the input flow to the tandem is Poisson.)

(4) The inequalities

$$
\lambda \mu_{1}<1, \quad \lambda \mu_{2}<1
$$

hold true. (It is the condition of absence of an overload.)

(5) There exist constants $\theta_{1}^{+} \in(0, \infty]$ and $\theta_{2}^{+} \in(0, \infty]$ such that the moment generating functions

$$
g_{j}(\theta)=\mathbf{E} e^{\theta \xi_{j}^{1}}<\infty, \quad \text { if } \theta<\theta_{j}^{+}, \quad j=1,2,
$$

and they are infinite for $\theta>\theta_{j}^{+}$if the boundary value $\theta_{j}^{+}$is finite.

We denote by $\theta_{j}, j=1,2$, positive solutions of the equations

$$
\theta_{j}=\lambda\left(g_{j}\left(\theta_{j}\right)-1\right)
$$

which will be used in the formulation of the main result. Let also

$$
g_{j}^{+}=\lim _{\theta \uparrow \theta_{j}^{+}} g_{j}(\theta), \quad j=1,2 .
$$

It is easy to verify that equation (2.5) has a unique positive solution if and only if

$$
g_{j}^{+} \geq 1+\frac{\theta_{j}^{+}}{\lambda}
$$

and $\mu_{j}>0$. For this purpose we observe that the function $\lambda\left[g_{j}\left(\theta_{j}\right)-1\right]$ is a strictly convex function of $\theta$ vanishing at the point $\theta=0$ and having the derivative $\lambda \mu_{j}$ which is less than 1 at 
this point.

In this simplest case, when the random variables $\xi_{j}^{i}$ have the exponential densities $\gamma_{j} e^{-\gamma_{j}^{x}}$, $j=1,2$, conditions (2.3) of absence of an overload is reduced to the condition $\lambda<\min \left\{\gamma_{1}, \gamma_{2}\right\}$. In this case, $\theta_{+}^{j}=\gamma_{j}, g_{j}^{+}=\infty$, and equation (2.5) has a positive solution for all $\lambda \in\left(0, \min \left\{\gamma_{1}, \gamma_{2}\right\}\right)$. The simplest example, when $g_{j}^{+}<\infty$ and so a solution of $(2.5)$ does not exist for small enough $\lambda$, is the case density proportional to $x^{-\alpha} \exp \{-\gamma x\}$, where $\gamma>0$ and $\alpha>1$.

Under conditions (1-5), the stationary mode of the considered system exists and is unique (see [14]). Now we will write a formula for the virtual waiting time $\omega$ in the steady state in terms of the sequence $\Xi$. For $t \geq 0$, let

$$
\nu(t)=\left\{\begin{array}{cl}
0, & \text { if } \tau^{1}>t \\
\max \left\{k: \sum_{i=1}^{k} \tau^{i} \leq t\right\}, & \text { if } \tau^{1} \leq t .
\end{array}\right.
$$

For $j=1,2$,

$$
\xi_{j}(t)=\left\{\begin{array}{cc}
0, & \text { if } \nu(t)=0 \\
\sum_{i=1}^{\nu(t)} \xi_{j}^{i}, & \text { if } \nu(t)>0 .
\end{array}\right.
$$

Each of the random processes $\xi_{j}(t), 0 \leq t<\infty, j=1,2$, is a homogeneous process with independent increments. Their trajectories are jump-wise (i.e., piecewise constant) nondecreasing, rightcontinuous functions. The virtual waiting time for the tandem can be expressed as

$$
\underset{v, s: 0 \leq v \leq s}{\omega}\left\{\xi_{2}(v)+\xi_{1}(s)-\xi_{1}(v-0)-s\right\} .
$$

Consequently, it is a functional of the two-dimensional homogeneous process

with independent increments.

$$
\boldsymbol{\xi}(t)=\left(\xi_{1}(t), \xi_{2}(t)\right)
$$

In the next section, we give arguments leading to relation (2.10). These arguments cannot be considered as mathematically rigorous because we deliberately avoid the use of the related strong definition of the virtual waiting time (cf. [1]) even though it can be applied to a mathematical derivation of this relation. A mathematical purist can treat formula (2.10) as the definition of the random variable we study in the paper.

Theorem 2.1: Under conditions (1-5),

$$
\lim _{x \rightarrow \infty} \frac{1}{x} \ln \operatorname{Pr}(\omega>x)=-\min \left\{\beta_{1}, \beta_{2}\right\},
$$

where $\beta_{1}$ and $\beta_{2}$ are defined by the relation ${ }^{3}$

$$
\beta_{j}=\left\{\begin{array}{cc}
\text { the positive root of }(2.5), & \text { if } g_{j}^{+} \geq 1+\frac{\theta_{j}^{+}}{\lambda}, \text { and } \mu_{j}>0, \\
\theta_{j}^{+}, & \text {if } g_{j}^{+}<1+\frac{\theta_{j}^{+}}{\lambda}, \text { and } \mu_{j}>0, \\
\infty, & \text { if } \mu_{j}=0 .
\end{array}\right.
$$

\footnotetext{
${ }^{3}$ In this formulation $\ln 0=\infty$.
} 
In the special case when the service times $\xi_{2}^{i} \equiv 0$, the quantity $\beta_{2}=\infty$. Thus, $\min \left\{\beta_{1}\right.$, $\left.\beta_{2}\right\}=\beta_{1}$. In this degenerate case, the tandem service reduces to the service by the first server only. It follows from Theorem 2.1 that

$$
\frac{1}{\alpha_{1}}=\beta_{1} \text { and } \frac{1}{\alpha_{2}}=\beta_{2}
$$

where $\alpha_{j}$ are the quantities defined by relations (1.3) for the case of the one server, and this theorem implies relation (1.4) formulated in $§ 1$. that

In the case of the exponential densities $\gamma_{j} e^{-\gamma_{j} x}$ of a random variables $\xi_{j}^{i}$, it is easy to see

$$
\beta_{j}=\gamma_{j}-\lambda
$$

if the inequalities $\lambda<\gamma_{j}, j=1,2$ hold true.

The construction developed below (see Note 5.5 for a precise formulation) permits us to describe the structure of most harmful fluctuations of the input flow and the service times of messages which produce the large values of the virtual service time at the moment 0 considered in the main Theorem 2.1. In the case $\beta_{1}<\beta_{2}$, they are fluctuations of density of the input flow and service times at the first device. In the case $\beta_{1}>\beta_{2}$, they are fluctuations of the density of the input flow and service times at the second device. There is an essential difference between the cases when condition (2.7) is satisfied and when it is not satisfied (for $j=1$, if $\beta_{1}<\beta_{2}$ and for $j=2$, if $\beta_{1}>\beta_{2}$ ). In the first case, the delay is mainly defined by the cumulative effect of fluctuations in a time interval of a length proportional to the delay and preceding the moment 0 . In the second case, the delay is defined by one message with the service time approximately equal to the delay of a message which arrives just before the moment 0 .

\section{The virtual waiting time}

We mentioned already in $\S 1$ that the virtual waiting time $\omega$ is the pure waiting time of a message excluding service, and it is served after all messages which entered the system earlier or the same moment. We define now the virtual waiting time of a message which enters the system at a moment $t$ as a value of the random process $\omega(t)$ which is a functional of a realization of the input flow of messages.

In queueing theory, an expression for the virtual waiting time in the system with a single device and FIFO discipline is well-known [1]. Consider a server with FIFO servicing discipline and assume that $\nu_{i}$ is the $i$ th moment of arrivaling messages, which requires time $\eta_{i}$ for service. The sequence $\left(\nu_{i}, \eta_{i}\right)_{i=-\infty}^{\infty}$ is the input flow to the system. Let ${ }^{4}$

$$
\eta(t)= \begin{cases}-\sum_{i: t \leq \nu_{i} \leq 0} \eta_{i}, & \text { if } t \leq 0 \\ \sum_{i: 0<\nu_{i}<t} \eta_{i}, & \text { if } t>0\end{cases}
$$

It is a left-continuous function. Then the virtual waiting time $\omega(t)$ of a virtual message arriving at a moment $t$ is

\footnotetext{
${ }^{4}$ Here and in the following, we interpret sums of an empty set of addends as 0 .
} 


$$
\omega(t)=\sup _{s \leq t}\{\eta(t+0)-\eta(s)-(t-s)\}
$$

Returning to the tandem system we recall that, in the equilibrium, the probability distribution of the virtual delay at $t$ does not depend on $t$ and so we can assume that $t=0$. Using the random variables introduced in $§ 2$ we define the sequence of moments

$$
\nu_{i}= \begin{cases}\sum_{k=0}^{i} \tau_{k}, & \text { if } i \geq 0 \\ -\sum_{k=i}^{-1} \tau_{k}, & \text { if } i<0\end{cases}
$$

as the sequence of the moments of arriving messages. As it is well known (see for example, [1], $\S 9$ ), this construction defines a stationary Poisson flow of moments. Now we put for $j=1,2$,

$$
\xi_{j}^{\prime}(t)= \begin{cases}-\sum_{i: t \leq \nu_{i} \leq 0} \xi_{j}^{i}, & \text { if } t \leq 0 \\ \sum_{i: 0<\nu_{i}<t} \xi_{j}^{i}, & \text { if } t>0\end{cases}
$$

The input flow to the first server is the flow $\left(\nu_{i}, \xi_{1}^{i}\right)_{i=-\infty}^{\infty}$. So, in terms of this flow we can express the virtual waiting time $\omega_{1}(t)$ at the first server for a virtual message arriving in the system at $t$ as

$$
\omega_{1}(t)=\sup _{s \leq t}\left\{\xi_{1}^{\prime}(t+0)-\xi_{1}^{\prime}(s)-(t-s)\right\}
$$

To establish a similar formula for the second server we need to describe the input flow to the second server. It is clear that the message which arrived at the first server at moment $\nu_{i}$ will enter the second server at the moment

$$
\sigma_{i}=\nu_{i}+\omega_{1}\left(\nu_{i}\right)
$$

It means that we have the input flow $\left(\sigma_{i}, \xi_{i}^{2}\right)_{i=-\infty}^{\infty}$ to the second server. We define similarly to (3.4) the process

$$
\widehat{\xi}_{2}(t)=\left\{\begin{array}{cl}
-\sum_{i: t \leq \sigma_{i} \leq 0} \xi_{2}^{i}, & \text { if } t \leq 0 \\
\sum_{i: 0<\sigma_{i}<t} \xi_{2}^{i}, & \text { if } t>0
\end{array}\right.
$$

Using relation (3.2) we can find the virtual waiting time at the second server for a virtual message arriving to the second server at moment $u$ as

$$
\widehat{\omega}(u)=\sup _{v \leq u}\left\{\widehat{\xi}_{2}(u+0)-\widehat{\xi}_{2}(v)-(u-v)\right\}
$$

If a virtual message enters the system at moment $t$ then it would arrive at the second server at moment

$$
u(t)=t+\omega_{1}(t)
$$


So its virtual waiting time at the second server is

$$
\omega_{2}(t)=\widehat{\omega}\left(t+\omega_{1}(t)\right) \underset{s \leq t+\omega_{1}(t)}{\sup }\left\{\widehat{\xi}_{2}\left(t+\omega_{1}(t)+0\right)-\widehat{\xi}_{2}(s)-\left(t+\omega_{1}(t)-s\right)\right\} .
$$

Comparing definitions (3.4) and (3.7) we see that for any two moments $v<t$,

$$
\xi_{2}^{\prime}(t+0)-\xi_{2}^{\prime}(v)=\widehat{\xi}_{2}\left(t+\omega_{1}(t)+0\right)-\widehat{\xi}_{2}\left(v+\omega_{1}(v)\right)
$$

The set of all moments $s=v+\omega_{1}(v)$ that for some $v$ coincides with the set of moments $\sigma_{i}$, at which the message enters the second device, is a discrete set only. But the maximized function in (3.10) is linear between these moments and so the upper bound in (3.10) is attained if we restrict it by these moments only. So using the change of variables $v \rightarrow s=v+\omega_{1}(v)$ we can rewrite $(3.10)$ as

$$
\omega_{2}(t)=\sup _{v \leq t}\left\{\xi_{2}^{\prime}(t+0)-\xi_{2}^{\prime}(v)-(t-v)-\left(\omega_{1}(t)-\omega_{1}(v)\right)\right\}
$$

Now using (3.5), we can evaluate the total virtual waiting time at both servers $\widetilde{\omega}(t)$ of a virtual message arrived in the tandem at moment $t$ as

$$
\begin{aligned}
& \tilde{\omega}(t)=\omega_{1}(t)+\omega_{2}(t)=\sup _{v \leq t}\left\{\xi_{2}^{\prime}(t+0)-\xi_{2}^{\prime}(v)-(t-v)+\omega_{1}(v)\right\} \\
& =\sup _{s, v: s \leq v \leq t}\left\{\left(\xi_{2}^{\prime}(t+0)-\xi_{2}^{\prime}(v)\right)+\left(\xi_{1}^{\prime}(v+0)-\xi_{1}^{\prime}(s)\right)-(t-s)\right\} .
\end{aligned}
$$

Letting $t=0$ and observing that $\xi_{2}^{\prime}(0+0)=0$ we find that

$$
\widetilde{\omega}=\sup _{s \leq v \leq 0}\left\{-\xi_{2}^{\prime}(v)+\left(\xi_{1}^{\prime}(v+0)-\xi_{1}^{\prime}(s)\right)+s\right\}
$$

It is more customary to consider random processes defined on the positive half-line, so it is convenient to make the transformation changing the sign of the time

$$
\left(t,\left(\xi_{1}^{\prime}(t), \xi_{2}^{\prime}(t)\right)\right) \rightarrow\left(-t,\left(-\xi_{1}^{\prime}(-t),-\xi_{2}^{\prime}(-t)\right)\right)
$$

This transformation transforms left-continuous functions to right-continuous functions. Applying this transformation to (3.14) we obtain that the random variables $\widetilde{\omega}$ and $\omega$ (see (2.10)) have the same distribution.

\section{Large deviations for processes with independent increments}

As was mentioned in $\S 1$, the proof of the main result of this paper is based on an application of a general theorem on large deviations for the processes with independent increments proved in [9]. In this section, we formulate only this theorem and prove some additional facts (Lemmas 4.1 and 4.2) which will be used together with the theorem in $\S 5$. First we recall the large deviations principle in its general formulation (see for example $[7,8]$ ).

Let $\mathscr{6}$ be a topological space and $\left\{P_{n}, n=1,2, \ldots\right\}$ be a sequence of probability measures on the $\sigma$-algebra of the Borel subsets of this space. The large deviations principle holds for the sequence $\left\{P_{n}\right\}$ with a rate function $I: \mathscr{S} \rightarrow[0, \infty]$ if for each Borel $^{5}$ set $B \subseteq \mathscr{G}$

\footnotetext{
${ }^{5}$ Here and in the following $\bar{B}$ is the closure of a set $B, B^{\circ}$ is its interior and $B^{c}$ is its complement.
} 


$$
\liminf _{n \rightarrow \infty} \frac{1}{n} \ln P_{n}(B) \geq \underset{\Re \in B^{\circ}}{\inf } I(\Re)
$$

and

$$
\limsup _{n \rightarrow \infty} \frac{1}{n} \ln P_{n}(B) \leq-\underset{\Re \in \bar{B}}{\inf I(\Re)}
$$

Lemma 4.1: Assume that a sequence $\left\{P_{n}\right\}$ is such that the large deviations principle holds for this sequence. Let $\mathfrak{H} \subseteq \mathscr{G}$ be a closed subset of $\mathscr{S}$ such that the measures $P_{n}$ are concentrated on this set, i.e.,

$$
P_{n}(\mathfrak{T G}) \equiv 1, \quad n=1,2, \ldots,
$$

For any subset $C \subseteq \mathfrak{H}$ we denote by $C_{\mathfrak{J} G}^{\circ}$ and $\bar{C}_{\mathfrak{H} G}$ the interior and the closure of the set $C$ with respect to the restriction of the topology in 96 to the set $\mathfrak{H} 6$. Then, for any Borel subset $B \subseteq \mathfrak{G}$,

and

$$
\liminf _{n \rightarrow \infty} \frac{1}{n} \ln P_{n}(B) \geq \underset{\Re \in(B \cap \mathcal{G})_{\mathcal{J}}^{\circ}}{-i n f} I(\Re),
$$

$$
\limsup _{n \rightarrow \infty} \frac{1}{n} \ln P_{n}(B) \leq-\frac{i n f}{\Re \in \overline{(B \cap \mathfrak{W})}_{H}} I(\Re) .
$$

Proof: It follows from the main condition (4.3) that

$$
P_{n}(B) \geq P_{n}\left((B \cap \mathcal{H})_{\mathfrak{H} G}^{\circ}\right)=P_{n}\left((B \cap \mathfrak{H})_{\mathcal{H}}^{\circ} \cup \mathcal{H} \mathcal{G}^{c}\right) .
$$

The set $(B \cap \mathcal{H})_{\mathcal{H} G}^{\circ} \cup \mathcal{J}^{C}$ is an open subset of $\mathscr{S}$ and so the estimate (4.1) and the identity (4.6) imply that

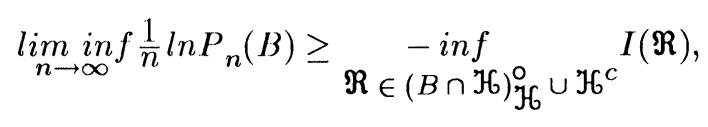

Applying the estimate (4.1) to $B=\mathcal{H}^{c}$ we find that

$$
I(\Re) \equiv \infty \text { for } \Re \in \mathfrak{H}^{c} \text {. }
$$

So the desired estimate (4.4) follows from (4.7). Since

$$
P_{n}(B) \equiv P_{n}(B \cap \mathfrak{H})
$$

and the set ${\overline{(B \cap \mathcal{H})_{\mathfrak{H}}}}_{\mathcal{G}}$ coincides with the closure of the set $B \cap \mathcal{H}$ in $\mathscr{S}$ the estimate (4.5) follows from (4.2) where $B$ is replaced by $B \cap \mathfrak{H}$.

Now we specify for the considered case all three objects introduced above: the topological space $\mathscr{G}$, the sequence of measures $\left\{P_{n}\right\}$ and the rate function $I$. We start with the space $\mathscr{G}$. Let $\mathfrak{R}(t)$ be a function of $t \in(-\infty, \infty)$ valued in Euclidean space $\mathbb{R}^{r}$. Recall that its variation on an interval $[s, u] \subset(-\infty, \infty)$ is equal to

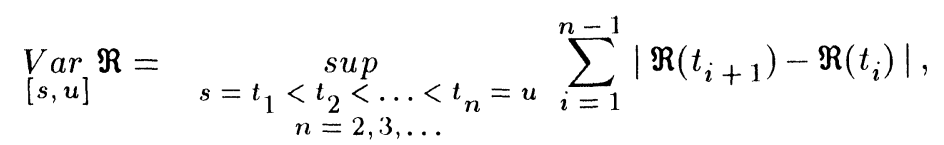

where the upper bound is taken over all partitions of the closed interval $[s, u]$. 
We consider a space $\mathscr{S}$ of functions $\Re:[0, \infty) \rightarrow \mathbb{R}^{r}$ with the following three properties:

The functions $\Re \in \mathscr{S}$ are functions with locally finite variation, i.e.,

$$
\underset{[0, t]}{\operatorname{Var}} \Re<\infty \text { for all } 0<t<\infty .
$$

(2) The functions $\Re \in \mathscr{C}$ are right-continuous at each point $t \geq 0$, i.e., for each $t \geq 0$ the limit $\lim _{u \downarrow t} \Re(u)$ exists and

$$
\Re(t)=\Re(t+0)=\lim _{u \downarrow t} \Re(u)
$$

(3) The limits

$$
v(\Re)=\lim _{t \rightarrow \infty} \frac{\Re(t)}{1+t}
$$

exist and are finite.

Sometimes, when it is convenient, we extend the functions $\Re \in 96$ by letting

$$
\Re(t)=0 \text { for }-\infty<t<0 .
$$

After such extension, the variation (4.10) does not depend on $s$ for $s<0 \leq u$ and we let

$$
\underset{[0-0, u]}{\operatorname{Var}} \Re=\underset{[s, u]}{\operatorname{Var}} \Re, \text { if } s<0 \leq u
$$

We shall treat $\mathscr{S}$ as a vector space with the natural definition of sums of its elements and their multiplication by a scalar. There is another useful interpretation of the space $\mathfrak{G}$. Observe that there is a one-to-one correspondence between functions $\mathfrak{R}:[0, \infty) \rightarrow \mathbb{R}^{r}$ with locally finite variation and $\sigma$-finite $\mathbb{R}^{r}$-valued measures $\mu_{\Re}$ on $[0, \infty)$. This correspondence is defined by the relation

$$
\Re(t)=\mu_{\Re}([0, t]), \quad t \geq 0
$$

Let $\Phi$ be the set of all continuous $\mathbb{R}^{r}$-valued functions $\phi(t), t \in \mathbb{R}$, with compact supports, i.e., those vanishing outside of a compact interval $\left[-T_{\phi}, T_{\phi}\right]$. For $\phi \in \Phi$ and $\mathfrak{R} \in \mathscr{G}$ we let

$$
J_{\phi}(\Re)=\int_{0}^{\infty} \phi(t) \Re(t) d t .
$$

(Here and in the following, $a b$ is the inner product of vectors $a, b \in \mathbb{R}^{r}$.) It is clear that for any fixed function $\phi \in \Phi$, we defined a linear functional $J_{\phi}$ on the space 96 . Now with each function $\phi \in \Phi$, we associate a function

$$
\widehat{\phi}(t)=\int_{t}^{\infty} \phi(u) d u .
$$

The function $\widehat{\phi}$ is continuous differentiable and vanishes for large $t$. Then,

$$
J_{\phi}(\Re)=\int_{0}^{\infty} \widehat{\phi}(t) \mu_{\Re}(d t) .
$$

For any number $T \geq 0$ we define a shift operator $S_{T}$ for the distance $T$ such that for any $\phi \in \Phi$, 


$$
S_{T} \phi(t)=\phi(t-T), \quad t \in \mathbb{R}^{1} .
$$

We define a topology on 96 by the system of pseudometrics

$$
\left.\rho_{\phi}(\Re, \mathfrak{R})=\sup _{n: n=0,1, \ldots}\left\{\frac{1}{1+n} \mid J_{S_{n} \phi}(\Re-\mathfrak{N})\right\}\right\}, \phi \in \Phi, \quad \mathfrak{R}, \mathfrak{N} \in \mathfrak{S} ;
$$

this means that a sequence $\left\{\mathfrak{R}_{N} \in \mathfrak{G}, N=1,2, \ldots\right\}$ converges to $\mathfrak{R} \in \mathfrak{G}$ if and only if

$$
\lim _{N \rightarrow \infty} \rho_{\phi}\left(\Re, \Re_{N}\right)=0 \text { for all } \phi \in \Phi .
$$

We shall call this topology a uniformly-weak topology. If for some $T>0$, the space $\mathscr{G}_{3}$ is a subspace of $\mathscr{S}$ consisting of all functions having a fixed restriction $3(t), t>T$, to the infinite interval $(T, \infty)$, then the induced topology on $\mathfrak{G}_{3}$ coincides with the usual weak topology for functions on the interval $[0, T]$.

The promised additional result absent in [9] is connected with another version of the topology in $\mathfrak{6}$. We consider a new system of pseudometrics defined as follows. Let $\phi \in \Phi$, and $\mathfrak{R}, \mathfrak{N} \in \mathfrak{G}$. The distance $\tilde{\rho}_{\phi}(\mathfrak{R}, \mathfrak{N})$ is

$$
\tilde{\rho}_{\phi}(\Re, \mathfrak{N})=\sup _{t \in[0, \infty)}\left\{\frac{1}{1+t}\left|J_{S_{t} \phi}(\Re-\mathfrak{N})\right|\right\} .
$$

Since $\tilde{\rho}_{\phi}(\Re, \mathfrak{R}) \geq \rho_{\phi}(\Re, \mathfrak{R})$, the topology generated by the pseudometrics $\tilde{\rho}_{\phi}(\mathfrak{R}, \mathfrak{N}), \phi \in \Phi$, is stronger than the uniformly-weak topology. The topology generated by the pseudometrics $\tilde{\rho}_{\phi}(\mathfrak{R}, \mathfrak{R}), \phi \in \Phi$, will be called the continuous uniformly-weak topology. We shall use the abbreviations "UW-topology" for the uniformly-weak topology and "CUW-topology" for the continuous uniformly-weak topology.

Lemma 4.2: The UW-topology and CUW-topology coincide in the restriction on the subset $\mathcal{Q} \subset \mathfrak{S}$ of all non-decreasing functions with non-negative components from $\mathscr{6}$.

Proof: We must show the UW-topology is not weaker than the CUW-topology.

In the first step we prove the following statement. For any function $\Re \in Q$ neighborhood $U_{\mathfrak{R}}^{\circ}$ of the point $\Re$ in the UW-topology and numbers $A, K<\infty$ such that

$$
|\mathfrak{N}(t)| \leq A+K t, \quad 0 \leq t<\infty, \mathfrak{N} \in U_{\mathfrak{R}}^{\circ} \cap \mathcal{Q} .
$$

We fix a function $\phi_{0} \in \Phi$ with non-negative components such that $\phi_{0}(t)=(1,1, \ldots, 1)$ for $0 \leq t \leq 2$ and take

$$
U_{\mathfrak{R}}^{\circ}=\left\{\mathfrak{N} \in \mathfrak{S}: \sup _{n: n=0,1, \ldots}\left\{\frac{1}{1+n}\left|J_{S_{n} \phi_{0}}(\Re-\mathfrak{N})\right|\right\}<1\right\} .
$$

The function $S_{[t]} \phi_{0}$, where $[t]$ is the integer part of the number $t$ is equal to $(1,1, \ldots, 1)$ on the whole interval $[t, t+1]$. Observe also that for any vector $a=\left(a_{1}, a_{2}, \ldots, a_{r}\right) \in \mathbb{R}^{r}$, its norm $|a| \leq\left|a_{1}\right|+\left|a_{2}\right|+\ldots+\left|a_{r}\right|$. So, for any function $\mathfrak{N} \in \mathcal{Q}$ and any $t \in[0, \infty)$,

$$
|\mathfrak{N}(t)| \leq \int_{t}^{t+1}|\mathfrak{N}(u)| d u \leq J_{S_{[t]} \phi_{0}}(\mathfrak{N})
$$

It follows from definitions $(4.17),(4.21)$ and (4.25) that for any function $\mathfrak{N} \in U_{\mathfrak{R}}^{\circ}$,

$$
J_{S_{[t]} \phi_{0}}(\mathfrak{\Re})=J_{S_{[t]} \phi_{0}}(\mathfrak{\Re}-\mathfrak{R})+J_{S_{[t]} \phi_{0}}(\mathfrak{R}) \leq 1+t+J_{S_{[t]} \phi_{0}}(\Re) .
$$


The properties (4.11) and (4.13) of the functions $\Re \in \mathscr{G}$ imply that for some constants $A^{\prime}=A^{\prime}(\Re)$ and $K^{\prime}=K^{\prime}(\Re)$,

$$
J_{S_{[t]} \phi_{0}}(\Re) \leq A^{\prime}+K^{\prime} t, \quad 0 \leq t<\infty .
$$

The desired estimate (4.24) follows from the estimates (4.26), (4.27) and (4.28) for $A=A^{\prime}+1$ and $K=K^{\prime}+1$.

Now we need to prove that for any $\Re \in Q$ and any open neighborhood $\widetilde{U}(\Re)$ of the point $\Re$ in the CUW-topology there exists an open neighborhood $U(\Re)$ of this point in the UW-topology such that $U(\Re) \cap \mathbb{Q} \subseteq \widetilde{U}(\Re) \cap \mathcal{Q}$. It is sufficient to consider the neighborhoods $\widetilde{U}(\Re)$ of the kind

$$
\widetilde{U}(\mathfrak{R})_{\phi, c}=\left\{\mathfrak{N}: \tilde{\rho}_{\phi}(\mathfrak{R}, \mathfrak{N})<c\right\}
$$

where $\phi \in \Phi, c>0$, since such neighborhoods generate the CUW-topology. Consider a number $\Delta$, $0<\Delta \leq 1$, such that $\Delta^{-1}$ is an integer and let

$$
U(\mathfrak{R})_{\phi, \Delta, c}=\left\{\mathfrak{N}: \rho_{S_{i \Delta} \phi}(\mathfrak{R}, \mathfrak{N})<c, \quad i=0,1, \ldots, \Delta^{-1}\right\} .
$$

We prove below that for any fixed $\phi \in \Phi$ and $c>0$ and for all sufficiently small $\Delta$,

$$
U(\Re)_{\phi, \Delta, c / 3} \cap U_{\mathfrak{R}}^{\circ} \cap \mathcal{Q} \subseteq \widetilde{U}(\Re)_{\phi, c} \cap \mathcal{Q}
$$

Since the set $U(\Re)_{\phi, \Delta, c / 3} \cap U_{\mathfrak{R}}^{\circ}$ is open in the UW-topology, the statement of the lemma will follow from this inclusion.

For any $t \in[0, \infty)$ we let $[t]_{\Delta}=k \Delta$, if $k \Delta \leq t<(k+1) \Delta$ and $k$ is an integer. Comparing definitions (4.21) and (4.23) we see that for $\mathfrak{N} \in U(\mathfrak{R})_{\phi, \Delta, c / 3}$ the distance

$$
\begin{aligned}
& \tilde{\rho}_{\phi}(\Re, \mathfrak{R}) \leq \sup _{i=0,1, \ldots, \Delta^{-1}}\left\{\rho_{S_{i \Delta} \phi}(\mathfrak{R}, \mathfrak{R})\right\} \\
& +\sup _{t \in[0, \infty)}\left\{\left|\frac{1}{1+t}\right| J_{S_{t^{\phi}}}(\Re-\mathfrak{N})\left|-\frac{1}{1+[t]}\right| J_{S_{[t]_{\Delta}{ }^{\phi}}(\Re-\mathfrak{N}) \mid} \mid\right\} \\
& \leq \frac{c}{3}+\sup _{t \in[0, \infty)}\left\{\left|\frac{1}{1+t}-\frac{1}{1+[t]}\right|\left|J_{S_{[t]_{\Delta^{\phi}}}}(\Re-\mathfrak{N})\right|\right\} \\
& +\sup _{t \in[0, \infty)}\left\{\frac{1}{1+t}\left|J_{S_{t} \phi}(\Re-\mathfrak{N})-J_{S_{[t]_{\Delta} \phi}}(\Re-\mathfrak{\Re})\right|\right\} \\
& \leq \frac{2 c}{3}+\sup _{t \in[0, \infty)}\left\{\frac{1}{1+t}\left|J_{S_{t} \phi}(\Re-\mathfrak{N})-J_{S_{[t]_{\Delta} \phi}}(\Re-\mathfrak{N})\right|\right\}
\end{aligned}
$$

It follows from the estimate (4.24) (which is also satisfied for $\mathfrak{N}$ replaced by $\mathfrak{R}$ ) and definitions (4.17), (4.20) that for $\mathfrak{N} \in U_{\mathfrak{R}}^{\circ}$,

$$
\begin{gathered}
\left|J_{S_{t} \phi}(\Re-\mathfrak{R})-J_{S_{[t]_{\Delta} \phi}}(\Re-\mathfrak{N})\right| \leq \int_{-\infty}^{\infty}\left|\phi(x-t)-\phi\left(x-[t]_{\Delta}\right)\right||\mathfrak{R}+\mathfrak{N}| d x \\
\leq 4 T_{\phi}\left(A+K\left(t+T_{\phi}\right)\right){ }_{x, x^{\prime}:\left|x-x^{\prime}\right| \leq \Delta}^{\max }\left|\phi(x)-\phi\left(x^{\prime}\right)\right|
\end{gathered}
$$

where $\left(-T_{\phi}, T_{\phi}\right)$ is an interval outside which the function $\phi$ vanishes. Since the function $\phi$ is a continuous function with a compact support, it follows from (4.33) that there exists a number $\Delta>0$ such that for all $t \in[0, \infty)$, 


$$
\frac{1}{1+t}\left|J_{S_{t} \phi}(\Re-\mathfrak{N})-J_{S_{[t]_{\Delta} \phi}}(\Re-\mathfrak{N})\right|<\frac{c}{3}
$$

So, it follows from (4.32) that

$$
\tilde{\rho}_{\phi}(\Re, \mathfrak{R}) \leq c
$$

which proves the desired inclusion (4.31).

Now we describe the measure $P_{n}$. First we recall the notion of a generalized Poisson process. Let $\pi$ be a non-negative measure on the space $\mathbb{R}^{r}$ such that the integral

$$
\int_{\mathbb{R}^{r}}|y| \pi(d y)<\infty
$$

We call a probability measure $P_{\pi}$ on Borel subsets of the space 96 a generalized Poisson measure with the jump measure $\pi$, if for any function $\phi \in \Phi$ the characteristic function is

$$
\int_{\mathscr{G}} \exp \left\{i J_{\phi}(\Re)\right\} P_{\pi}(d \Re)=\exp \left\{\int_{0}^{\infty} \int_{\mathbb{R}^{r}}(\exp \{i y \widehat{\phi}(t)\}-1) \pi(d y) d t\right\}
$$

(see notation (4.18)). Heuristically it means that we consider a time-homogeneous Poisson process such that the probability of a jump of $y \in A$ in the time interval of length $d t$ is equal to $\pi(A) d t$, if $\pi(A)<\infty$.

The existence of the measure $P_{\pi}$ on $\mathscr{G}$ follows from a well-known statement that, under condition (4.36), almost all realizations of a process with independent increments are functions with a locally bounded variation (cf. [11], ch. IV, $\S 1$ ), and from the strong law of large numbers which guarantees the existence of the limits (4.13). It follows from definition (4.37) that

$$
v(\Re)=m, \quad P_{\pi^{-}} \text {a.s. }
$$

where the mean value

$$
m=\int_{\mathbb{R}^{r}} y \pi(d y) \in \mathbb{R}^{r}
$$

Let $T_{n}: \mathscr{G} \rightarrow \mathscr{S}$ be a transformation

$$
T_{n}: \Re(t) \rightarrow \Re_{n}(t)=\frac{1}{n} \Re(n t) .
$$

It is easy to verify that the conditions (1-3) included in the definition of the space 9 hold for the function $\Re_{n}(t)$, if they do for $\Re(t)$. Let $P_{\pi}^{n}$ be a measure on $\mathscr{G}$ induced by the transformation $T_{n}$ from the measure $P_{\pi}$. It is easy to see that $P_{\pi}^{n}$ defines again a generalized Poisson process with the jump measure

$$
\pi_{n}(A)=n \pi(n A)
$$

Now we define the rate function. We suppose that for some $a>0$

$$
\int_{\mathbb{R}^{r}}\left(e^{a|y|}-1\right) \pi(d y)<\infty .
$$


This inequality implies the condition (4.36). Let

$$
q(\theta)=\int_{\mathbb{R}^{r}}\left(e^{\theta y}-1\right) \pi(d y), \quad \theta \in \mathbb{R}^{r},
$$

and let $\Theta_{\pi}$ be set of points $\theta \in \mathbb{R}^{r}$ for which $q(\theta)<\infty$. It is easy to verify that $q(\theta)$ is a convex function of $\theta \in \mathbb{R}^{r}$ and so $\Theta_{\pi}$ is a convex set. Let

$$
\Lambda_{a}(x)=\sup _{\theta \in \Theta_{\pi}}\{\theta x-q(\theta)\}, \quad x \in \mathbb{R}^{r}
$$

The function $\Lambda_{a}(x)$ is the Legendre transformation of the function $q$. It is a convex non-negative (because $\theta x-q(\theta)=0$, if $\theta=0$ ) function of $x$ valued in $[0, \infty]$. Let $\Theta_{\pi}^{\circ}$ be a set of all interior points of the set $\Theta_{\pi}$ which is non-empty, because of the condition (4.42). It is clear that $q(\theta)$ is smooth in the domain $\Theta_{\pi}^{\circ}$. If for some $x \in \mathbb{R}^{r}$ there exists $\theta_{x} \in \Theta_{\pi}^{\circ}$ such that the value of the gradient

$$
\nabla q\left(\theta_{x}\right)=x
$$

then

$$
\Lambda_{a}(x)=\theta_{x} x-q\left(\theta_{x}\right)
$$

(see [18], §26). It is clear that $\nabla q(0)=m$ and so

$$
\Lambda_{a}(m)=0
$$

In addition, let

$$
\Lambda_{s i}(x)=\sup _{\theta \in \Theta_{\pi}} \theta x
$$

Observe that

$$
\Lambda_{s i}(x)=\lim _{\gamma \rightarrow \infty} \frac{1}{\gamma} \Lambda_{a}(\gamma x), \quad x \in \mathbb{R}^{r}
$$

It holds true that $\theta x-q(x)=-\infty$, if $\theta \notin \Theta_{\pi}$ and so $\gamma^{-1} \Lambda_{a}(\gamma x) \leq \sup _{\theta \in \Theta_{\pi}} \theta x$, for any $x$ and $\gamma$. On the other hand, for any fixed $\theta \in \Theta_{\pi}$ and $x$ the quantity $\gamma^{-1}(\theta \gamma x-q(\theta)) \rightarrow \theta x$ as $\gamma \rightarrow \infty$, and hence the upper bound in (4.49) can not be greater than $\lim _{\gamma \rightarrow \infty} \frac{1}{\gamma} \Lambda_{a}(\gamma x)$. The function $\Lambda_{s i}(x)$ can be interpreted as the Legendre transformation of the function

$$
\psi(\theta)= \begin{cases}0, & \theta \in \Theta_{\pi} \\ \infty, & \theta \notin \Theta_{\pi}\end{cases}
$$

Again (see [18], §13), $\Lambda_{s i}(x)$ is a convex non-negative function of $x \in \mathbb{R}^{r}$. It is linear on any ray $\{\lambda x, 0 \leq \lambda<\infty\}, x \in \mathbb{R}^{r}$. This function is called a support function of the set $\Theta_{\pi}$. If $r=1$, then

$$
\Lambda_{s i}(x)=\left\{\begin{array}{cl}
x \sup \left\{\theta: \theta \in \Theta_{\pi}\right\}, & \text { if } x>0 \\
x \inf \left\{\theta: \theta \in \Theta_{\pi}\right\}, & \text { if } x<0 \\
0, & \text { if } x=0
\end{array}\right.
$$


Observe that for any $x_{1}, x_{2} \in \mathbb{R}^{r}$ and $\theta \in \Theta_{\pi}$,

$$
\theta\left(x_{1}+x_{2}\right)=\theta x_{1}+\theta x_{2} \leq \Lambda_{s i}\left(x_{1}\right)+\Lambda_{s i}\left(x_{2}\right) .
$$

Therefore, the function $\Lambda_{s i}(x)$ is subadditive:

$$
\Lambda_{s i}\left(x_{1}+x_{2}\right) \leq \Lambda_{s i}\left(x_{1}\right)+\Lambda_{s i}\left(x_{2}\right), \quad x_{1}, x_{2} \in \mathbb{R}^{r} \text {. }
$$

Any function $\Re \in \mathscr{G}$ can be represented in a unique way as

$$
\Re=\Re_{a}+\Re_{s i},
$$

where $\Re_{a}$ is an absolutely continuous function

$$
\Re_{a}(t)=\int_{0}^{t} \dot{\Re}_{a}(u) d u
$$

and $\Re_{s i}$ is singular, i.e., a function such that the corresponding measure $\mu_{\Re_{s i}}$ is a singular measure with respect to the Lebesgue measure. Let

$$
I_{a}\left(\Re_{a}\right)=\int_{0}^{\infty} \Lambda_{a}\left(\dot{\Re}_{a}(u)\right) d u
$$

(the integral has sense because $\Lambda_{a} \geq 0$ ) and

$$
I_{s i}\left(\Re_{s i}\right)=\int_{0}^{\infty} \Lambda_{s i}\left(\dot{\Re}_{s i}(u)\right) d u .
$$

Of course, the derivative $\dot{\mathfrak{R}}_{s i}(u)$ does not exist and hence the last integral (4.57) needs a corresponding interpretation. Let $\mathscr{P}$ be the system of all finite partitions $\Pi=\left\{-\infty<t_{0}<t_{1}<\right.$ $\left.\ldots<t_{n}<\infty\right\}, n=1,2, \ldots$. Let

$$
I_{s i}^{\Pi}\left(\Re_{s i}\right)=\sum_{k=1}^{n} \Lambda_{s i}\left(\Re_{s i}\left(t_{k}\right)-\Re_{s i}\left(t_{k-1}\right)\right)
$$

and

$$
I_{s i}\left(\Re_{s i}\right)=\sup _{\Pi \in \mathscr{\rho}}\left\{I_{s i}^{\Pi}\left(\Re_{s i}\right)\right\}
$$

We shall say that a partition $\mathrm{II}^{\prime}=\left\{-\infty<t_{0}^{\prime}<t_{1}^{\prime}<\ldots<t_{n^{\prime}}^{\prime}<\infty\right\}$ is a subpartition on the partition $\Pi$ if each point $t_{i}^{\prime}$ coincides with one of the points $t_{k}$. It follows from non-negativity and subadditivity of the function $\Lambda_{s i}$ that, if $\mathrm{II}^{\prime}$ is a subpartition of $\Pi$, then

$$
I_{s i}^{\Pi^{\prime}}\left(\Re_{s i}\right) \leq I_{s i}^{\Pi}\left(\Re_{s i}\right) .
$$

So we can also interpret $I_{s i}\left(\Re_{s i}\right)$ as the limit of $I_{s i}^{\Pi I}\left(\Re_{s i}\right)$ with respect to the partial order on the set $\mathscr{P}$ defined with the aid of the subpartitions.

In the case $r=1$, the function $\Re_{s i}(t)$ can be represented in a unique way as a sum

$$
\Re_{s i}(t)=\Re_{s i}^{+}(t)+\Re_{s i}^{-}(t),
$$

where $\Re_{s i}^{+}(t)$ is a non-decreasing function and $\Re_{s i}^{-}(t)$ is a non-increasing function of $t \in[0, \infty)$, 
and supports of the measures $\mu_{\mathfrak{R}_{s i}^{+}}$and $\mu_{\mathfrak{R}_{s i}^{-}}$are disjoint. Then it follows from (4.51) that

$$
I_{s i}\left(\Re_{s i}\right)=\Re_{s i}^{+}(\infty) \sup \left\{\theta: \theta \in \Theta_{\pi}\right\}+\Re_{s i}^{-}(\infty) \inf \left\{\theta: \theta \in \Theta_{\pi}\right\}
$$

Here $\Re_{s i}^{ \pm}(\infty)=\lim _{u \rightarrow \infty}, \Re_{s i}^{ \pm}(u)$, with $0 \infty=0$.

Let

$$
I(\Re)=\int_{0}^{\infty} \Lambda_{a}\left(\dot{\Re}_{a}(u)\right) d u+\int_{0}^{\infty} \Lambda_{s i}\left(\dot{\Re}_{s i}(u)\right) d u .
$$

(Here $a+\infty=\infty$ for any $a \in(-\infty, \infty])$.

Theorem 4.3: If condition (4.42) holds true then the sequence of the probability measures $\left\{P_{\pi}^{n}, \quad n=1,2, \ldots\right\}$ satisfies the large deviations principle with the rate function I defined in (4.63).

For the case $r=1$ and the processes given in a finite interval, this theorem has been proved by Lynch and Sethuraman in [15].

The proof of Theorem 4.3 is given in [9].

We shall use also the following fact which is also proved in [9], Lemma 6.4. The rate function

$$
I(\Re)=\infty, \quad \text { if } v(\Re) \neq m
$$

\section{The proof of Theorem 2.1}

This proof is based on Theorem 4.3 for the case $r=2$ when the space of trajectories 9 consists of functions $\Re(t)=\left(\Re_{1}(t), \Re_{2}(t)\right)$ valued in $\mathbb{R}^{2}$. The spaces of possible values of components $\Re_{1}(t)$ and $\Re_{2}(t)$ which are defined by the construction in $\S 4$ for the case $r=1$ will be denoted $\mathscr{G}_{1}$ and $\mathscr{G}_{2}$.

We assume that the jump measure $\pi$ defining the Poisson measure $P_{\pi}$ (see (4.37)), is the product measure

$$
\pi=\lambda\left(\pi_{1} \otimes \pi_{2}\right)
$$

where the parameter $\lambda$ was introduced in $\S 2$ and $\pi_{j}, j=1,2$, are the probability distributions of the random variables $\xi_{j}^{i}$ introduced in the same section. It is easy to see that the random process $\xi(t)$ defined by the relations (2.9) and (2.11) coincides with the Poisson process with the jump measure $\pi$. Consider (cf. (2.10)) the following functional defined on the space 96

$$
\omega(\Re)=\sup _{s, v: 0 \leq v \leq s}\left\{\Re_{2}(v)+\Re_{1}(s)-\Re_{1}(v-0)-s\right\} .
$$

Since the functions $\Re \in \mathscr{S}$ have a locally bounded variation (see (4.11)), the left-sided limits $\mathfrak{R}(v-0)$ exist and this functional is well defined. In terms of the normalized process with the distribution $P_{\pi}^{n}$, for any integer $n$, the probability studied for Theorem 2.1 (see $(2.12),(2.10)$ ) is

where

$$
\operatorname{Pr}(\omega>n)=P_{\pi}^{n}(\mathcal{A})
$$

$$
\mathcal{A}=\{\mathfrak{R}: \omega(\mathfrak{R})>1\}
$$


We let

$$
\begin{gathered}
\mathcal{Q}_{j}=\left\{\Re_{j} \in \mathfrak{G}: 0 \leq \Re_{j}(t) \leq \Re_{j}(u), \text { for all } t<u\right\}, \quad j=1,2, \\
\mathbb{Q}=\left\{\mathfrak{R}=\left(\Re_{1}, \mathfrak{R}_{2}\right) \in \mathfrak{S}_{:}: \mathfrak{R}_{1} \in \mathcal{Q}_{1}, \mathfrak{R}_{2} \in \mathcal{Q}_{2}\right\}
\end{gathered}
$$

$\mathcal{Q}$ is the subset of $\mathscr{S}$ consisting of all pairs of non-decreasing and non-negative functions. It is obvious that $\mathcal{Q}$ is a closed set in the uniformly-weak topology. Since we assumed in $\S 2$ that the random variables $\xi_{j}^{i}$ are non-negative, the measures $P_{\pi}^{n}$ are concentrated on the set $\mathcal{Q}$. So we can apply the principle of large deviations in the formulation of Lemma 4.1 for $\mathfrak{H} 6=\mathbb{Q}$ for which we will consider the interior $(\mathcal{A} \cap \mathcal{Q})_{\mathbb{Q}}^{\circ}$ and the closure $\overline{(\mathcal{A} \cap \mathcal{Q})}{ }_{\mathbb{Q}}$ with respect to the restriction of the uniformly-weak topology to $\mathcal{Q}$ used in the formulation of this lemma.

Lemma 5.1: Let

$$
\omega^{0}(\Re)=\sup _{0 \leq v \leq s}\left\{\max \left\{\Re_{2}(v-0)-\Re_{1}(v-0), \Re_{2}(v)-\Re_{1}(v)\right\}+\Re_{1}(s)-s\right\}
$$

Then,

$$
(\mathcal{A} \cap \mathbb{Q})_{\mathbb{Q}}^{\circ} \supseteq\left\{\Re: \omega^{0}(\Re)>1\right\}
$$

Proof: Assume that $\Re \in Q$ is such that $\omega^{0}(\Re)>1$. There are two cases. For some $0 \leq v \leq s$, either

$$
\Re_{2}(v)-\Re_{1}(v)+\Re_{1}(s)-s>1
$$

or

$$
\Re_{2}(v-0)-\Re_{1}(v-0)+\Re_{1}(s)-s>1
$$

In case (5.8), since $\Re$ is a right continuous function, there exists an $\epsilon=\epsilon(\Re)>0$ such that for any small $\epsilon^{\prime}>0$,

$$
\Re_{2}(t)-\Re_{1}(t)>1+3 \epsilon-\Re_{1}(s)+s, \text { if } v \leq t \leq v+\epsilon^{\prime} .
$$

Take a function $\phi=\left(\phi_{1}, \phi_{2}\right) \in \Phi$ such that $\phi_{1}(t) \equiv-\phi_{2}(t)$, where the function $\phi_{2}$ is non-negative, vanishes outside the interval $\left(v, v+\epsilon^{\prime}\right)$ and is such that its integral over this interval equals 1 . It follows from estimate (5.10) and the definition of the topology that there exists a neighborhood $U(\mathfrak{R})$ of the point $\mathfrak{R}$ such that (see $(4.17)$ ) for any function $\mathfrak{N}=\left(\mathfrak{N}_{1}, \mathfrak{N}_{2}\right) \in U(\mathfrak{R}) \cap \mathcal{Q}$, it holds true that

$$
J_{\phi}(\mathfrak{\Re}) \geq J_{\phi}(\Re)-\epsilon \geq 1+2 \epsilon-\Re_{1}(s)+s
$$

Since

$$
J_{\phi}(\mathfrak{N})=\int_{v}^{v+\epsilon^{\prime}}\left(\mathfrak{N}_{2}(t)-\mathfrak{N}_{1}(t)\right) \phi_{2}(t) d t
$$

it follows from (5.11) that there exists a point $v^{\prime}=v^{\prime}(\mathfrak{N}) \in\left(v, v+\epsilon^{\prime}\right)$ such that

$$
\mathfrak{N}_{2}\left(v^{\prime}\right)-\mathfrak{\Re}_{1}\left(v^{\prime}-0\right) \geq \mathfrak{\Re}_{2}\left(v^{\prime}\right)-\mathfrak{N}_{1}\left(v^{\prime}\right) \geq 1+2 \epsilon-\mathfrak{R}_{1}(s)+s .
$$


In a similar way, assuming that the neighborhood $U(\Re)$ and the number $\epsilon^{\prime}>0$ are small, we can find a value $s^{\prime} \geq s+\epsilon^{\prime} \geq v^{\prime}$ such that for $\mathfrak{N} \in U(\Re) \cap \mathcal{Q}$,

$$
\mathfrak{R}_{1}\left(s^{\prime}\right)-s^{\prime} \geq \mathfrak{R}_{1}(s)-s-\epsilon .
$$

It follows from (5.13) and (5.14) that

$$
\omega(\mathfrak{N})>1
$$

The case (5.9) is similar. Under the assumption that $v>0$, there exists $\epsilon=\epsilon(\Re)>0$ such that for any small $\epsilon^{\prime}>0$,

$$
\Re_{2}(t)-\Re_{1}(t)>1+3 \epsilon-\Re_{1}(s)+s, \text { if } v>t \geq v-\epsilon^{\prime}
$$

A construction analogous to the previous one derives the estimate (5.15) from inequality (5.16). In the special case, when $v=0$ in $(5.9), \Re(0-0)=0$, condition (5.9) degenerates to the inequality $\Re_{1}(s)-s>1$ and so that for some $\epsilon>0$ and any function $\mathfrak{N} \in \mathbb{Q}$,

$$
\mathfrak{N}_{2}(0)-\mathfrak{N}_{1}(0-0)+\mathfrak{R}_{1}(s)-s>1+2 \epsilon
$$

We can use estimate (5.14) again, and together with (5.17) it will also imply the inequality (5.15) for this last case. It follows from inequality (5.15) that $\mathfrak{R}$ is an interior point of the set $\mathcal{A} \cap \mathcal{Q}$.

Note 5.1: One can verify that

$$
(\mathcal{A} \cap \mathcal{Q})_{\mathbb{Q}}^{\circ}=\left\{\Re: \omega^{0}(\Re)>1\right\}
$$

although unnecessary for our objectives. That some points of the set of the trajectories $\mathcal{A} \cap \mathcal{Q}$ need not be its interior points can be explained in terms of our queueing problem. Assume that at time $t$, when the lines to each server are short, a message enters the system requesting service times $\xi_{1}$ and $\xi_{2}$ to the respective channels, and that $\xi_{1}$ and $\xi_{2}$ are large. Then, the service delay is close to $\xi_{2}+\xi_{2}$. Alternatively, suppose at time $t-\Delta$ (with small $\Delta>0$ ), a message enters the system and requires only service time $\xi_{2}$ at the second channel, whereas another message entering the system at time $t$ requires service time $\xi_{1}$ only at the first channel. Then both messages can be served in parallel, and consequently, the service delay is close to $\max \left\{\xi_{1}, \xi_{2}\right\}$. But the corresponding trajectories of the input flow are close in the weak topologies.

For $\mathfrak{R}=\left(\mathfrak{R}_{1}, \boldsymbol{R}_{2}\right) \in \mathfrak{G}$ we let $v(\mathfrak{R})=\left(v_{1}\left(\boldsymbol{R}_{1}\right), v_{2}\left(\mathfrak{R}_{2}\right)\right)$ (see $\left.(4.13)\right)$, and then let

$$
w(\mathfrak{R})=\max \left\{v_{1}\left(\boldsymbol{R}_{1}\right), v_{2}\left(\boldsymbol{R}_{2}\right)\right\} .
$$

Lemma 5.2: Let the set $\widehat{A}$ consist of all functions $\Re \in \mathcal{Q}$ for which either there exist points $s \leq v$ such that

$$
\Re_{2}(v)+\Re_{1}(s)-\Re_{1}(v-0) \geq s+1
$$

or

$$
w(\Re) \geq 1
$$

Then the following inclusion holds true:

$$
\overline{(\mathcal{A} \cap Q)}_{\mathbb{Q}} \subseteq \widehat{A}
$$


Proof: Let a function $\Re=\left(\Re_{1}, \Re_{2}\right) \in \overline{(\mathcal{A} \cap \mathbb{Q})}_{\mathcal{Q}}$. There exists a sequence $\Re_{n}=\left\{\Re_{1, n}\right.$, $\left.\mathfrak{R}_{2, n}\right) \in \mathcal{A} \cap \mathbb{Q}$ which converges to $\mathfrak{R}$. Note that if $t \in[0, \infty)$ is a point of continuity of the function $\mathfrak{R}_{i}, i=1,2$, then $\mathfrak{R}_{i, n}(t) \rightarrow \mathfrak{R}_{i}(t)$. It follows from definitions (5.2) and (5.4) that there exists a sequence of pairs of points $\left(v_{n} \leq s_{n}\right)$ from $[0, \infty)$ such that

$$
\Re_{2, n}\left(v_{n}\right)+\Re_{1, n}\left(s_{n}\right)-\Re_{1, n}\left(v_{n}-0\right)>s_{n}+1 .
$$

There are three possibilities for the sequence of the pairs $\left(v_{n} \leq s_{n}\right)$.

In the first case, the sequence $\left\{s_{n}\right\}$ is bounded. Then, using compactness arguments, we suppose without loss of generality that the sequence $\left\{\Re_{n}\right\}$ is such that $v_{n} \rightarrow v$ and $s_{n} \rightarrow s$ for some $v \leq s<\infty$. Assume that points $s+\tau, v+\sigma$ and $v-v$ are continuity points of the functions $\mathfrak{R}_{1}$ and $\mathfrak{R}_{2}$ where $\tau, \sigma$ and $\nu$ are positive. Then $s_{n}<s+\tau$ and $v-\nu<v_{n}<v+\sigma$ for large $n$ and so the inequalities

$\Re_{2, n}(v+\sigma)+\Re_{1, n}(s+\tau)-\Re_{1, n}(v-\nu) \geq \Re_{2, n}\left(v_{n}\right)+\Re_{1, n}\left(s_{n}\right)-\Re_{1, n}\left(v_{n}-0\right)>s_{n}+1$

hold true. On the other hand,

$$
\Re_{2 n}(v+\sigma)+\Re_{1, n}(s+\tau)-\Re_{1, n}(v-\nu) \rightarrow \Re_{2}(v+\sigma)+\Re_{1}(s+\tau)-\Re_{1}(v-\nu)
$$

as $n \rightarrow \infty$. Therefore,

$$
\mathfrak{R}_{2}(v+\sigma)+\mathfrak{R}_{1}(s+\tau)-\mathfrak{R}_{1}(v-\nu) \geq s+1 .
$$

Because $\sigma, \tau$ and $\nu$ can be chosen arbitrarily small, and the functions $\Re_{1}$ and $\mathfrak{R}_{2}$ are rightcontinuous, the inequality

$$
\Re_{2}(v)+\Re_{1}(s)-\Re_{1}(v-0) \geq s+1
$$

is valid.

Now we assume that the sequence $\left\{v_{n}\right\}$ is bounded but the sequence $\left\{s_{n}\right\}$ is unbounded and so we can suppose without loss of generality that $s_{n} \rightarrow \infty$ and $v_{n} \rightarrow v<\infty$ as $n \rightarrow \infty$. Let $\sigma>0$ and $\nu>0$ be chosen in such a way that $v+\sigma$ is a continuity point of the function $\Re_{2}$ and $v-\nu$ is a continuity point of the function $\Re_{1}$. Then, as above we verify that

$$
\underset{n \rightarrow \infty}{\limsup }\left(\Re_{2, n}\left(v_{n}\right)-\Re_{1, n}\left(v_{n}-0\right)\right) \leq \Re_{2}(v+\sigma)-\Re_{1}(v-\nu)<\infty
$$

So it follows from inequality (5.23) that

$$
\liminf _{n \rightarrow \infty} \frac{\Re_{1, n}\left(s_{n}\right)}{1+s_{n}} \geq 1
$$

Now let $\phi_{0}$ be a non-negative function vanishing outside the interval $(0,1)$ and such that its integral over this interval is equal to 1 . Because the function $\Re_{1, n}$ is non-decreasing, it follows from (5.29) that

$$
\limsup _{n \rightarrow \infty} \frac{\int_{-\infty}^{\infty} \Re_{1, n}(u) \phi_{0}\left(u-s_{n}\right) d u}{1+s_{n}} \geq \limsup _{n \rightarrow \infty} \frac{\Re_{1, n}\left(s_{n}\right)}{1+s_{n}} .
$$

Lemma 4.2 implies that the sequence $\mathfrak{R}_{n} \rightarrow \mathfrak{R}$ in CUW-topology also. Hence (see (4.23)), $\tilde{\rho}_{\phi}\left(\Re_{n}, \Re\right) \rightarrow 0$, where we let $\phi=\left(\phi_{0}, 0\right)$, and inequality (5.30) implies that 


$$
\limsup _{n \rightarrow \infty} \frac{\int_{\infty}^{\infty} \Re_{1, n}(u) \phi_{0}\left(u-s_{n}\right) d u}{1+s_{n}}=\limsup _{n \rightarrow \infty} \frac{\int_{-\infty}^{\infty} \Re_{1}(u) \phi_{0}\left(u-s_{n}\right) d u}{1+s_{n}} .
$$

Due to definition (4.13) we see that there exists the limit

$$
\lim _{n \rightarrow \infty} \frac{\int_{-\infty}^{\infty} \Re_{1}(u) \phi_{0}\left(u-s_{n}\right) d u}{1+s_{n}}=v\left(\Re_{1}\right)
$$

The estimates $(5.29),(5.30),(5.31),(5.32)$ imply that $v\left(\Re_{1}\right) \geq 1$ and so we arrived at the desired condition (5.21).

The third case is when both sequences $v_{n}$ and $s_{n}$ are unbounded and therefore it is reasonable to assume that $v_{n} \rightarrow \infty$ and $s_{n} \rightarrow \infty$ as $n \rightarrow \infty$. Using the compactness arguments we can also assume without loss of generality that the limit

$$
\alpha=\lim _{n \rightarrow \infty} \frac{1+v_{n}}{1+s_{n}}
$$

exists, where $0 \leq \alpha \leq 1$. Applying again estimates (5.30), (5.31) and (5.32) we find that

$$
\underset{n \rightarrow \infty}{\limsup } \frac{\Re_{1}\left(s_{n}\right)}{1+s_{n}} \leq v\left(\Re_{1}\right)
$$

Consider the function $\phi=\left(-\phi_{0}(\bullet-1), \phi_{0}\right) \in \Phi$ where the function $\phi_{0}$ was introduced above and $\phi_{0}(\bullet-1)$ is its shift vanishing outside of the interval $(-1,0)$. Then, using the monotonicity of the functions $\Re_{1, n}, \Re_{2, n}$ we find that

$$
J_{S_{v_{n}} \phi_{0}}\left(\Re_{n}\right)=\int_{-\infty}^{\infty} \phi_{0}(t)\left(\Re_{2, n}\left(t+v_{n}\right)-\Re_{1, n}\left(t+v_{n}-1\right)\right) d t \geq \Re_{2}\left(v_{n}\right)-\Re_{1}\left(v_{n}-0\right) .
$$

Similarly to (5.31), we derive from Lemma 4.2 that

$$
\limsup _{n \rightarrow \infty} \frac{J_{S_{v_{n}} \phi_{0}}\left(\Re_{n}\right)}{1+v_{n}}=\limsup _{n \rightarrow \infty} \frac{J_{S_{v_{n}} \phi_{0}}(\Re)}{1+v_{n}} .
$$

Similarly to (5.32), we derive that

$$
\lim _{n \rightarrow \infty} \frac{J_{S_{v_{n}} \phi_{0}}(\Re)}{1+v_{n}}=v\left(\Re_{2}\right)-v\left(\Re_{1}\right) .
$$

Returning to inequality (5.23) and then using the estimates (5.33)-(5.37) we find that

$$
\begin{aligned}
& 1 \leq \liminf _{n \rightarrow \infty} \frac{\Re_{2, n}\left(v_{n}\right)-\Re_{1, n}\left(v_{n}-0\right)+\Re_{1, n}\left(s_{n}\right)}{1+s_{n}} \\
& \leq \limsup _{n \rightarrow \infty}\left(\frac{\Re_{2, n}\left(v_{n}\right)-\Re_{1, n}\left(v_{n}-0\right)}{1+v_{n}} \frac{1+v_{n}}{1+s_{n}}\right)+\underset{n \rightarrow \infty}{\limsup } \frac{\Re_{1, n}\left(s_{n}\right)}{1+s_{n}} \\
& \leq \underset{n \rightarrow \infty}{\limsup }\left(\frac{J_{S_{v_{n}} \phi_{0}}{ }^{(\Re)}}{1+v_{n}} \frac{1+v_{n}}{1+s_{n}}\right)+\underset{n \rightarrow \infty}{\limsup _{n \rightarrow n}} \frac{\Re_{1, n}\left(s_{n}\right)}{1+s_{n}} \\
& \leq\left(v\left(\Re_{2}\right)-v\left(\Re_{1}\right)\right) \alpha+v\left(\Re_{1}\right)=v\left(x_{2}\right) \alpha+v\left(x_{1}\right)(1-\alpha) \leq \max \left\{v\left(\Re_{1}\right), v\left(\Re_{2}\right)\right\}=w(\Re) .
\end{aligned}
$$


We have arrived at the desired condition (5.21).

Note 5.2: It is possible to verify that

$$
{\overline{(\mathcal{A} \cap \mathcal{Q})_{\mathcal{Q}}}}=\widehat{\mathcal{A}}
$$

Again the latter is unnecessary for the rest of the paper.

Now we want to describe some properties of the rate function (4.63) with respect to the jump measure (5.1). Observe that in this case the domain $\Theta_{\pi}$, in which the moment generating function $q(\theta)$ is finite, is such that

$$
\Theta_{\pi}=\Theta_{\pi_{1}} \times \Theta_{\pi_{2}}
$$

where

$$
\Theta_{\pi_{j}}=\left\{\begin{array}{cc}
\left(-\infty, \theta_{j}^{+}\right], & \text {if } g_{j}^{+}<\infty \\
\left(-\infty, \theta_{j}^{+}\right), & \text {if } g_{j}^{+}=\infty, \quad j=1,2,
\end{array}\right.
$$

and the quantities $\theta_{j}^{+}$and $g_{j}^{+}$are defined by relations (2.4) and (2.6). We shall use the following notation for the rate functions of the components of the process being considered (cf. (4.43), (4.44)):

where

$$
\Lambda_{a, j}\left(v_{j}\right)=\sup _{\theta_{j} \in(-\infty, \infty)}\left\{v_{j} \theta_{j}-q_{j}\left(\theta_{j}\right)\right\}, \quad j=1,2
$$

$$
q_{1}\left(\theta_{1}\right)=\lambda\left(g_{1}\left(\theta_{1}\right)-1\right)=q\left(\theta_{1}, 0\right), \quad q_{2}\left(\theta_{2}\right)=\lambda\left(g_{2}\left(\theta_{2}\right)-1\right)=q\left(0, \theta_{2}\right)
$$

and the moment generating functions

$$
g_{j}\left(\theta_{j}\right)=\int_{\mathbb{R}^{1}} \exp \left\{\theta_{j} x_{j}\right\} \pi_{j}\left(d x_{j}\right), \quad j=1,2,
$$

were introduced by relation (2.4). We let also (cf. (4.63), (4.51) and (5.41))

$$
I_{j}\left(\Re_{j}\right)=\int_{0}^{\infty} \Lambda_{a, j}\left(\dot{\Re}_{a, j}\right) d t+\theta_{j}^{+} \Re_{s i, j}(\infty), \quad \Re_{j} \in \mathcal{Q}_{j}, \quad j=1,2
$$

where $\Re_{a, j}, \Re_{s i, j}$ are absolutely continuous and singular components of the functions $\Re_{j}$ with $\mathfrak{R}_{j}(\infty)=\lim _{u \rightarrow \infty} \mathfrak{R}_{j}(u), j=1,2$. follows:

Lemma 5.3: For any singular function $\mathfrak{R}=\left(\Re_{1}, \Re_{2}\right) \in \mathbb{Q}$ (see $\left.\S 4\right)$, the rate function is as

$$
I(\Re)=I_{s i}(\Re)=\theta_{1}^{+} \Re_{1}(\infty)+\theta_{2}^{+} \Re_{2}(\infty)
$$

Proof: Applying definition (4.48) to (5.40) and (5.41), we find that for any $x=\left(x_{1}, x_{2}\right) \in \mathbb{R}^{2}$,

$$
\Lambda_{s i}(x)=\theta_{1}^{+} x_{1}+\theta_{2}^{+} x_{2}
$$


The statement of the lemma follows immediately from definitions (4.57) and (4.63).

Lemma 5.4: For any $y_{1} \geq 0$,

$$
\inf _{w \in(-\infty, \infty)} \Lambda_{a}\left(y_{1}, w\right)=\Lambda_{a, 1}\left(y_{1}\right)=\Lambda_{a}\left(y_{1}, w\left(y_{1}\right)\right)
$$

where under the assumption that the measure $\pi_{1}$ is such that it is not concentrated at the point 0 ,

$$
w\left(y_{1}\right)=\left\{\begin{array}{cl}
\lambda \mu_{2} g_{1}\left(\widehat{\theta}_{1}\left(y_{1}\right)\right), & \text { if } y_{1}>0 \\
0, & \text { if } y_{1}=0
\end{array}\right.
$$

the mean values $\mu_{j}$ are defined by relation $(2.2)$, and the value $\widehat{\theta}_{1}\left(y_{1}\right)$ is such that

$$
\left.\Lambda_{a, 1}\left(y_{1}\right)=\sup _{\theta_{1}}\left\{y_{1} \theta_{1}-q_{1}\left(\theta_{1}\right)\right\}=y_{1} \widehat{\theta}_{1}\left(y_{1}\right)-q_{1}\left(\widehat{\theta}_{1}\left(y_{1}\right)\right)\right)
$$

In the special case, when the measure $\pi_{1}$ is concentrated in the point 0 , relation (5.48) holds true for

$$
w\left(y_{1}\right)=\mu_{2}
$$

Proof: It is obvious from definition (4.44) that for any $w \in \mathbb{R}^{1}$,

$$
\Lambda_{a}\left(y_{1}, w\right)=\sup _{\left(\theta_{1}, \theta_{2}\right) \in \Theta_{\pi}}\left\{y_{1} \theta_{1}+w \theta_{2}-q\left(\theta_{1}, \theta_{2}\right)\right\} \geq \sup _{\theta_{1}}\left\{y_{1} \theta_{1}-q\left(\theta_{1}, 0\right)\right\}=\Lambda_{a, 1}\left(y_{1}\right),
$$

so it is sufficient to show that

$$
\Lambda_{a, 1}\left(y_{1}\right)=\Lambda_{a}\left(y_{1}, w\left(y_{1}\right)\right)
$$

Observe that

$$
q\left(\theta_{1}, 0\right)=q_{1}\left(\theta_{1}\right)=\lambda \int_{-\infty}^{\infty}\left(e^{\theta_{1} y}-1\right) \pi_{1}(d y)
$$

Consider the case when the measure $\pi_{1}$ is not concentrated at the point 0 and assume that $y_{1}>0$. Then it is clear that $q_{1}\left(\theta_{1}\right)$ is a continuous function of $\theta_{1}$ convergent to $-\lambda$ as $\theta_{1} \rightarrow-\infty$. Furthermore, the derivative $q_{1}^{\prime}\left(\theta_{1}\right) \rightarrow \infty$ as $\theta_{1} \rightarrow \infty$, if $\theta_{1}^{+}=\infty$. In the case when $\theta_{1}^{+}<\infty$ but $g_{1}^{+}=\infty$, the value $q_{1}\left(\theta_{1}\right) \rightarrow \infty$, as $\theta_{1} \rightarrow \theta_{1}^{+}$. It follows that for $y_{1}>0$ the strictly concave continuous function $y_{1} \theta_{1}-q_{1}\left(\theta_{1}\right)$ of $\theta_{1} \in \Theta_{\pi_{1}}$ has a unique maximal point inside the set $\Theta_{\pi_{1}}$. So the values $\widehat{\theta}_{1}\left(y_{1}\right)$ and $w\left(y_{1}\right)$ are well-defined by the relations (5.50) and (5.49). In the case when $\theta_{1}^{+}<\infty, g_{1}^{+}<\infty$, the value $\widehat{\theta}_{1}\left(y_{1}\right)$ can be equal to the end point $\theta_{1}^{+}$of the interval $\Theta_{\pi_{1}}$.

It follows from definitions (4.44), (5.1) and (2.4) that

$$
q(\theta)=q\left(\theta_{1}, \theta_{2}\right)=\lambda\left(g_{1}\left(\theta_{1}\right) g_{2}\left(\theta_{2}\right)-1\right)
$$

Assume that $\widehat{\theta}_{1}\left(y_{1}\right)<\theta_{1}^{+}$. Then the derivative $q_{1}^{\prime}\left(\widehat{\theta}_{1}\left(y_{1}\right)\right)=y_{1}$. So, using definitions (5.50), (2.2) and (2.4) we find that the value of the gradient is

$$
\left.\nabla q\left(\theta_{q}, \theta_{2}\right)\right|_{\theta_{1}=\widehat{\theta}_{1}\left(y_{1}\right), \theta_{2}=0}=\left(y_{1}, w\left(y_{1}\right)\right) \text {. }
$$

$\left(\widehat{\theta}_{1}\left(y_{1}\right), 0\right)$ is an interior point of the set $\Theta_{\pi}$ (see (5.40)) and thus the desired equation (5.53) 
follows from the relations (4.45) and (4.46). In the case when $\widehat{\theta}_{1}\left(y_{1}\right)=\theta_{1}^{+}$, the point $\left(\widehat{\theta}_{1}, 0\right)$ is an interior point of an edge of the set $\Theta_{\pi}$. Let

$$
\phi\left(\theta_{1}, \theta_{2}\right)=y_{1} \theta_{1}+w\left(y_{1}\right) \theta_{2}-q\left(\theta_{1}, \theta_{2}\right), \quad\left(\theta_{1}, \theta_{2}\right) \in \Theta_{\pi},
$$

and consider the partial derivatives of this strictly concave function at the point $\left(\widehat{\theta}_{1}, 0\right)$. Its derivative at $\theta_{2}$ is equal to 0 , its left derivative at $\theta_{1}$ is equal to $y_{1}-\left(q_{1}\right)_{\text {left }}^{\prime}\left(\hat{\theta}_{1}\left(y_{1}\right)\right) \leq 0$. So the point $\left(\widehat{\theta}_{1}, 0\right)$ is a point of a local maximum of the function $\phi$. But for a strictly concave function, any point of its local maximum is the point of its global maximum. It proves the desired relation (5.53) for this case also.

If $y_{1}=0$, a direct computation shows that $\Lambda_{a, 1}(0)=\Lambda_{a}(0,0)=\lambda$.

In this case, where $\pi_{1}$ is concentrated at $x_{1}=0$,

$$
\inf _{w \in(-\infty, \infty)} \Lambda_{a}\left(y_{1}, w\right)=\Lambda_{a}\left(y_{1}, \mu_{2}\right)= \begin{cases}0, & \text { if } y_{1}=0 \\ \infty, & \text { if } y_{1}>0\end{cases}
$$

This proves the lemma.

Note 5.3: The first of (5.48) can also be obtained in a roundabout way by a use of the principle of large deviations. Indeed, the left term in (5.48) actually describes the asymptotics of the probability of the event $\left\{\Re:\left|\Re_{1}(n)-n y_{1}\right|<\epsilon\right\}$ for small $\epsilon>0$ defined by the twodimensional Poisson process with the jump measure $\pi$. But the same probability can be treated as the probability defined by the one-dimensional Poisson process with the jump measure $\pi_{1}$ and rate function $\Lambda_{a, 1}$. The second equality in (5.48) can be explained in the following way. The condition $\left\{\Re:\left|\Re_{1}(n)-n y_{1}\right|<\epsilon\right\}$ changes the density of arriving messages somehow but the mean value of the service times of any message in the second device stays equal to $\mu_{2}$.

Note 5.4: The statement which is obtained from Lemma 5.4 by interchanging the indices 1 and 2 is of course also valid. The same is true for Lemmas 5.5 and 5.6 and relation (5.59).

The next formula easily follows from relations (5.42), (5.43) and (5.44) under the condition $\mu_{1}>0$ :

$$
\Lambda_{a, 1}(w)=\left\{\begin{array}{cc}
\theta_{w, 1} w-q_{1}\left(\theta_{w, 1}\right), & \text { if } \theta<w<\infty \text { and } \theta_{1}^{+}=\infty \text { or } q_{1}^{\prime}\left(\theta_{1}^{+}\right)=\infty, \\
\theta_{1}^{+} w-q_{1}\left(\theta_{1}^{+}\right), & \text {and if } 0<w \leq q_{1}^{\prime}\left(\theta_{1}^{+}\right), \theta_{1}^{+}<\infty, \text { and } q_{1}^{\prime}\left(\theta_{1}^{+}\right)<\infty \\
\pi([0, \infty)), & \text { if } w>q_{1}^{\prime}\left(\theta_{1}^{+}\right) . \\
\infty, & \text { if } w=0, \\
& \text { if } w<0,
\end{array}\right.
$$

where $q_{1}^{\prime}$ is the derivative of the function $q_{1}$, and $\theta_{w, 1}$ is the unique solution of the equation

$$
q_{1}^{\prime}\left(\theta_{w, 1}\right)=w
$$

Lemma 5.5: Assume that $\mu_{1}>0$. For any $u>0, a \geq 0$ let

$$
\begin{gathered}
\mathcal{Y}_{1}(u, a)=\left\{\Re_{1} \in \mathcal{Q}_{1}: \Re_{1}(u)=a\right\}, \\
\mathcal{Y}(u, a)=\left\{\Re=\left(\Re_{1}, \Re_{2}\right) \in \mathbb{Q}: \Re_{1}(u)=a\right\} .
\end{gathered}
$$


Consider the function $\mathfrak{\Im}=\left(\Im_{1}, \Im_{2}\right)$ such that

$$
\begin{gathered}
\Im_{1}(t)=\left\{\begin{array}{cc}
\frac{a}{u} t, & \text { if } 0 \leq t \leq u, \\
a+m_{1}(t-u), & \text { if } u<t<\infty,
\end{array}\right. \\
\Im_{2}(t)=\left\{\begin{array}{cc}
t w\left(\frac{a}{u}\right), & \text { if } 0 \leq t \leq u, \\
u w\left(\frac{a}{u}\right)+m_{2}(t-u), & \text { if } u<t<\infty,
\end{array}\right.
\end{gathered}
$$

where the function $w\left(y_{1}\right)$ was introduced in Lemma 5.4 and the mean value $m=\left(m_{1}, m_{2}\right)($ see (4.39)). Then,

$$
\inf _{\Re \in \mathcal{Y}(u, a)} I(\Re)=I(\Im)=\inf _{\Re_{1} \in \mathcal{Y}_{1}(u, a)} I_{1}(\Re)=I_{1}\left(\Im_{1}\right)=u \Lambda_{a, 1}\left(\frac{a}{u}\right) .
$$

Proof: The relation

$$
I(\Im)=I_{1}\left(\Im_{1}\right)=u \Lambda_{a, 1}\left(\frac{a}{u}\right)
$$

follows immediately from relations (4.63), (5.48) and (5.45). So we have to prove only the inequality

$$
I(\Re) \geq I_{1}\left(\Re_{1}\right) \geq u \Lambda_{a, 1}\left(\frac{a}{u}\right)
$$

for any $\Re \in \mathcal{Y}(u, a)$. For this purpose we observe that it follows from equality (5.48) and the convexity and non-negativity of the function $\Lambda_{a, 1}$ that for any absolutely continuous function $\Re \in \mathcal{Y}(u, a)$,

$$
\begin{aligned}
I(\Re)= & \int_{0}^{\infty} \Lambda_{a}(\dot{\Re}(t)) d t \geq I_{1}\left(\Re_{1}\right) \geq \int_{0}^{u} \Lambda_{a, 1}\left(\dot{\Re}_{1}(t)\right) d t \\
\geq u \Lambda_{a, 1}\left(\frac{1}{u} \int_{0}^{u} \dot{\Re}_{1}(t) d t\right) & =u \Lambda_{a, 1}\left(\frac{a}{u}\right) .
\end{aligned}
$$

It follows from definition (4.63), Lemmas 5.3 and 5.4 and the positivity of the function $\Lambda_{a, 1}$ that for any function $\Re \in Q$,

$$
\begin{aligned}
& I(\Re)=\int_{0}^{\infty} \Lambda_{a}\left(\dot{\Re}_{a}(t)\right) d t+\theta_{1}^{+} \Re_{s i, 1}(\infty)+\theta_{2}^{+} \Re_{s i, 2}(\infty) \\
& \geq I_{1}\left(\Re_{1}\right) \geq \int_{0}^{u} \Lambda_{a, 1}\left(\dot{\Re}_{a, 1}(t)\right) d t+\theta_{1}^{+} \Re_{s i, 1}(u) .
\end{aligned}
$$

Fixing a function $\Re \in \mathcal{Y}(u, a)$, consider the sequence $\mathfrak{R}_{1, N}$ of absolutely continuous functions with values in $\mathbb{R}^{1}$ such that their derivatives are

where

$$
\dot{\mathfrak{R}}_{1, N}(t)=\left\{\begin{array}{cc}
\alpha_{N} \dot{\mathfrak{R}}_{a, 1}\left(\alpha_{N} t\right), & \text { if } t<u-\frac{1}{N}, \\
N \Re_{s i, 1}(u), & \text { if } u-\frac{1}{N}<t \leq u
\end{array}\right.
$$

$$
\alpha_{N}=\frac{u}{u-\frac{1}{N}}
$$


(We assume that $N>u^{-1}$.) It is clear that $\Re_{1, N}(u)=\Re_{1}(u)=a$ and

$$
\int_{0}^{u} \Lambda_{a, 1}\left(\dot{\mathfrak{R}}_{1}^{N}(t)\right) d t=\left(\alpha_{N}\right)^{-1} \int_{0}^{u} \Lambda_{a, 1}\left(\alpha_{N} \dot{\mathfrak{R}}_{a, 1}(t)\right) d t+\frac{1}{N} \Lambda_{a, 1}\left(N \Re_{s i, 1}(u)\right) .
$$

The quantity $\alpha_{N} \rightarrow 1$, as $N \rightarrow \infty$. It follows from equation (5.59) that the function $\Lambda_{a, 1}(x)$ is a continuous function of $x \geq 0$. Relation (4.49) applied for the function $\Lambda_{s i, 1}$ shows that for $x>0$,

$$
\lim _{\gamma \rightarrow \infty} \frac{\Lambda_{a, 1}(\gamma x)}{\gamma}=\theta_{1}^{+} x
$$

Using these facts we derive from (5.70) that

$$
\lim _{N \rightarrow \infty} \int_{0}^{u} \Lambda_{a, 1}\left(\dot{\Re}_{1}^{N}(t)\right) d t=\int_{0}^{u} \Lambda_{a, 1}\left(\dot{\Re}_{a, 1}(t)\right) d t+\theta_{1}^{+} \Re_{s i, 1}(u)
$$

and so the desired estimate (5.65) follows from (5.66) and (5.67).

Lemma 5.6: Assume that $\mu_{1}>0$, and that for any $z \geq 0$,

$$
\begin{gathered}
\mathscr{B}_{1}(z)=\left\{\Re_{1} \in \mathcal{Q}_{1}: \sup _{u \geq 0}\left\{\Re_{1}(u)-u\right\} \geq z\right\}, \\
\mathscr{B}(z)=\left\{\Re=\left(\Re_{1}, \Re_{2}\right) \in \mathbb{Q}: \sup _{u \geq 0}\left\{\Re_{1}(u)-u\right\} \geq z\right\} .
\end{gathered}
$$

Then,

$$
\begin{gathered}
\inf _{\Re \in \mathscr{B}(z)} I(\Re)=z \beta_{1}=I\left(\Im_{z}\right), \\
i n f \underset{\mathfrak{R}_{1} \in \mathfrak{B}_{1}(z)}{I_{1}\left(\Re_{1}\right)=z \beta_{1}=I_{1}\left(\Im_{z, 1}\right),}
\end{gathered}
$$

where the value $\beta_{1}$ is defined by (2.13) and the function $\mathfrak{\Im}_{z}=\left(\mathfrak{\Im}_{z, 1}, \Im_{z, 2}\right)$ is defined by the formula (5.62) with

$$
u=\frac{z}{q_{1}^{\prime}\left(\theta_{1}\right)-1}, \quad a=q_{1}^{\prime}\left(\theta_{1}\right) u
$$

when condition (2.7) for $j=1$ is met and so the value $\theta_{1}$ (see (2.5)) is defined,and for the opposite case,

$$
\begin{gathered}
\Im_{z, 1}(t)=z+m_{1} t, \\
\Im_{z, 2}(t)=m_{2} t, \quad 0 \leq t<\infty .
\end{gathered}
$$

Proof: The proofs of both equalities (5.74) are completely similar, so we prove only the first one. First we prove the inequality

$$
\inf _{\Re \in \mathscr{B}(z)} I(\Re) \geq z \beta_{1} .
$$

We introduce the class of sets

$$
\mathfrak{C}_{u}=\left\{\Re \in \mathbb{Q}: \Re_{1}(u) \geq u+z\right\} .
$$

Comparing definitions (5.73) and (5.78) we see that for any function $\Re \in \mathfrak{B}(z)$ either $\Re \in C_{u}$ for some $u \geq 0$ or $v_{1}\left(\Re_{1}\right) \geq 1>m_{1}$. In the last case, $I(\Re)=\infty$ (see $\left.(4.64)\right)$, so we see that 


$$
\inf _{\Re \in \mathscr{B}(z)} I(\Re) \geq \inf _{u \geq 0} \inf _{\Re \in \mathcal{C}_{u}} I_{a}\left(\Re_{a}\right)
$$

Using this relation, the inequality $\Re_{1}(u) \geq \Re_{a, 1}(u)$, the statement (5.63) of Lemma 5.5 , and the monotonicity of the function $\Lambda_{a, 1}(x)$ in $x \geq 1$, we deduce that

$$
\begin{gathered}
\inf _{\Re \in \mathscr{B}(z)} I(\Re) \geq \inf _{\substack{u: 0<u<\infty \\
x: x \geq u+z}} u \Lambda_{a, 1}\left(\frac{x}{u}\right) \\
=\inf _{u: 0<u<\infty} u \Lambda_{a, 1}\left(\frac{u+z}{u}\right)=\inf _{w: 1<w<\infty} \frac{z \Lambda_{a, 1}(w)}{w-1} .
\end{gathered}
$$

(In the last equality we let $w=u^{-1}(u+z)$ ).

While investigating the minimum in (5.80) we treat two different cases. In the first case, $\theta_{1}^{+}=\infty$ or $\theta_{1}^{+}<\infty$, but $q_{1}^{\prime}\left(\theta_{1}^{+}\right)=\infty$, and so we can use the first line in (5.59) for all $w>1$. We find that the derivative is

$$
\begin{aligned}
& \frac{\partial}{\partial w}\left(\frac{\Lambda_{a, 1}(w)}{w-1}\right)=\frac{1}{(w-1)^{2}}\left(\frac{\partial \Lambda_{a, 1}(w)}{\partial w}(w-1)-\Lambda_{a, 1}(w)\right) \\
& =\frac{1}{(w-1)^{2}}\left(\theta_{w, 1}(w-1)-\Lambda_{a, 1}(w)\right)=\frac{q_{1}\left(\theta_{w, 1}\right)-\theta_{w, 1}}{(w-1)^{2}}
\end{aligned}
$$

It follows from definition (5.59) that the derivative $\left(\theta_{w, 1}\right)_{w}^{\prime}=\left(q_{1}^{\prime \prime}\left(\theta_{w, 1}\right)\right)^{-1}>0$ and so $\theta_{w, 1}$ is an increasing function of $w$.

Now we consider two subcases. In the first subcase, condition (2.7) with $j=1$ is met and so there exists a unique positive root $\theta_{1}$ of the equation $q\left(\theta_{1}\right)=\theta_{1}$ (cf. (2.5)). It follows from the description of the behavior of the function $q_{1}(\theta)=\lambda\left(g_{1}(\theta)-1\right)$ given in $\S 2$ that the function $q_{1}\left(\theta_{w, 1}\right)-\theta_{w, 1}$ vanishes at the point $\bar{w}_{1}$. It is negative for $w<\bar{w}_{1}$ and positive for $w>\bar{w}_{1}$. It implies that the function $(w-1)^{-1} \Lambda_{a, 1}(w), 1<w<\infty$ has its minimum at the point $\bar{w}_{1}$, if, of course, $\bar{w}_{1}>1$. The last inequality is valid, since the function $q_{1}(\theta)-\theta$ takes its minimal value when $q_{1}^{\prime}(w)=1$ and is negative at this point. Since in the considered case $\theta_{1}=\beta_{1}$, we obtain by direct computation on the right-hand side of (5.80) that (see (2.13) and (5.59))

$$
\inf _{\Re \in \mathscr{B}(z)} I(\Re) \geq z \frac{\Lambda_{a, 1}\left(\bar{w}_{1}\right)}{\bar{w}_{1}-1}=z \frac{\theta_{1} \bar{w}_{1}-q_{1}\left(\theta_{1}\right)}{\bar{w}_{1}-1}=z \theta_{1}=z \beta_{1}
$$

in the considered subcase.

The second subcase is when condition (2.7) with $j=1$ is not met. Then the difference $q_{1}\left(\theta_{w, 1}\right)-\theta_{w, 1}$ is negative for all $w>1$. The solution, $\theta_{w, 1}$ of $(5.60)$ approaches $\theta_{1}^{+}$, as $w \rightarrow \infty$. The function $(w-1)^{-1} \Lambda_{a, 1}(w)$ is decreasing, and, therefore, from (5.59) and definition (2.13) it follows that

$$
\underset{1<w<\infty}{i n f} \frac{z \Lambda_{a, 1}(w)}{w-1}=\lim _{w \rightarrow \infty} \frac{z \Lambda_{a, 1}(w)}{w-1}=z \theta_{1}^{+}=z \beta_{1}
$$

in this subcase.

The second case is when $\theta_{1}^{+}<\infty$, and $q_{1}^{\prime}\left(\theta_{1}^{+}\right)<\infty$. Evidently from definition (4.43), in this case $q_{1}\left(\theta_{1}^{+}\right)<\infty$ also. Then relation (5.81) can be used for $w<q^{\prime}\left(\theta_{1}^{+}\right)$. For $w>q^{\prime}\left(\theta_{1}^{+}\right)$we find, using the second line in (5.59), that

$$
\frac{\partial}{\partial w}\left(\frac{\Lambda_{a, 1}(w)}{w-1}\right)=\frac{q_{1}\left(\theta_{1}^{+}\right)-\theta_{1}^{+}}{(w-1)^{2}}<0 .
$$


Again, the lower bound of the function $(w-1)^{-1} \Lambda_{a, 1}(w)$ is reached for $w \rightarrow \infty$ and

$$
\inf _{\mathfrak{R} \in \mathfrak{B}_{(z)}} I(\Re) \geq z \lim _{w \rightarrow \infty} \frac{\theta_{1}^{+} w-q\left(\theta_{1}^{+}\right)}{w-1}=z \theta_{1}^{+}=z \beta_{1} .
$$

We prove the desired estimate (5.77) for all possible cases.

Since $\Im_{z} \in \mathfrak{B}(z)$ we complete the proof by verifying

$$
z \beta_{1}=I\left(\Im_{z}\right)
$$

In the case when condition (2.7) is satisfied, equation (5.86) follows from the relations (5.63) and (5.75) and a direct computation similar to (5.81). In the opposite case, (5.86) follows from the relations (5.46) and (4.47).

Lemma 5.7: Assume that $\mu_{1}>0$ and $\mu_{2}>0$. For the set of trajectories $\widehat{\mathcal{A}}$ defined in Lemma 5.2 ,

$$
\inf I(\Re)=\min \left\{\beta_{1}, \beta_{2}\right\}=I(\mathfrak{N})
$$

$\mathfrak{R} \in \hat{\mathcal{A}}$

where when $\beta_{1} \leq \beta_{2}$, the function $\mathfrak{N}$ is the function $\mathfrak{\Im}_{\{z, 1\}}$, with $z=1$, described in Lemma 5.6 . When $\beta_{1} \geq \beta_{2}$, the function $\mathfrak{N}$ is obtained by the same construction but with the interchanged indices 1 and 2 .

Proof: Observe that if $w(\Re) \geq 1$ (see (5.21)) then either $m_{1}<v_{1}(\Re)$ or $m_{2}<v_{2}(\Re)$. It follows from relation (4.64) that

$$
I(\Re)=\infty, \text { if } w(\Re) \geq 1
$$

So,

where

$$
\inf _{\Re \in \widehat{\mathcal{A}}} I(\Re)=\inf _{\Re \in \tilde{\mathcal{A}}} I(\Re),
$$

$$
\tilde{\mathcal{A}}=\cup_{v, s: 0 \leq v \leq s}\left\{\Re \in \mathcal{Q}: \Re_{2}(v)+\Re_{1}(s)-\Re_{1}(v-0) \geq s+1\right\} .
$$

First we prove the inequality

Let for any $\gamma \in \mathbb{R}^{1}$ and $0 \leq v \leq s$,

$$
\inf _{\Re \in \tilde{\mathcal{A}}} I(\Re) \geq \min \left\{\beta_{1}, \beta_{2}\right\} .
$$

$$
\mathscr{B}_{v, s, \gamma}=\left\{\Re \in \mathbb{Q}: \Re_{2}(v)-v \geq \gamma, \Re_{1}(s)-\Re_{1}(v-0)-(s-v) \geq 1-\gamma\right\} .
$$

Then,

$$
\tilde{\mathcal{A}}=\cup_{v, s, \gamma: 0 \leq v \leq s, \gamma \in \mathbb{R}^{1 \mathfrak{B}_{v, s, \gamma}}}
$$

Consider a function $\mathfrak{R}=\left(\Re_{1}, \mathfrak{R}_{2}\right) \in \mathfrak{B}_{v, s, \gamma}$ and let $\mathfrak{R}_{a, j}, \mathfrak{R}_{s i, j}, j=1,2$ be the absolutely continuous and singular parts of the corresponding functions. Using definition (4.63) and relations (5.46) and (5.48) (also with the indices interchanged.) we find that 


$$
\begin{array}{r}
I(\Re)=\int_{0}^{\infty} \Lambda_{a}\left(\dot{\mathfrak{R}}_{a, 1}(t), \dot{\mathfrak{R}}_{a, 2}(t)\right) d t+\theta_{1}^{+} \Re_{s i, 1}(\infty)+\theta_{2}^{+} \Re_{s i, 2}(\infty) \\
\geq \int_{0}^{v} \Lambda_{a, 2}\left(\dot{\Re}_{a, 2}\right) d t+\theta_{2}^{+} \Re_{s i, 2}(v)+\int_{v}^{s} \Lambda_{a, 1}\left(\dot{\Re}_{a, 1}\right) d t+\theta_{1}^{+}\left(\Re_{s i, 1}(s)-\Re_{s i, 1}(v-0)\right) .
\end{array}
$$

Let

$$
\widehat{\Re}_{1}(t)=\left\{\begin{array}{cc}
\Re_{1}(t+v), & \text { if } 0 \leq t<s-v, \\
\Re_{1}(s)+m_{1}(t-s+v), & \text { if } s-v \leq t<\infty .
\end{array}\right.
$$

It follows from definition (5.45) that

$$
I_{1}\left(\widehat{\Re}_{1}\right)=\int_{v}^{s} \Lambda_{a, 1}\left(\dot{\Re}_{a, 1}\right) d t+\theta_{1}^{+}\left(\Re_{s i, 1}(s)-\Re_{s i, 1}(v-0)\right) .
$$

From the comparison of conditions (5.92) and (5.73) we see that for $\gamma \leq 1$ the function $\widehat{\Re}_{1} \in \mathfrak{B}_{1}(1-\gamma)$. Thus Lemma 5.6 implies that

$$
I_{1}\left(\widehat{\Re}_{1}\right) \geq \max (1-\gamma, 0) \beta_{1} .
$$

Consider also the function

$$
\widehat{\Re}_{2}(t)=\left\{\begin{array}{cc}
\Re_{2}(t), & \text { if } 0 \leq t \leq v \\
\mathfrak{R}_{2}(v)+m_{2}(t-v) & \text { if } v<t<\infty
\end{array}\right.
$$

It follows from definition (5.45) that

$$
I_{2}\left(\widehat{\Re}_{2}\right)=\int_{0}^{v} \Lambda_{a, 2}\left(\dot{\Re}_{a, 2}\right) d t+\theta_{2}^{+} \Re_{s i, 2}(v) .
$$

We can again (cf. (5.97)) apply Lemma 5.6 but now with the indices 1 and 2 interchanged and obtain that

$$
I_{2}\left(\Re_{2}\right) \geq \max (\gamma, 0) \beta_{2}
$$

The estimates (5.94), (5.97) and (5.100) imply that for any $\Re \in \tilde{\mathcal{A}}$

$$
I(\Re) \geq \max (\gamma, 0) \beta_{1}+\max (1-\gamma, 0) \beta_{2} \geq \min \left\{\beta_{1}, \beta_{2}\right\},
$$

and this proves the desired estimate (5.91).

In the case $\beta_{1} \leq \beta_{2}$, the equality

$$
I\left(\Im_{\{1,1\}}\right)=\beta_{1}
$$

follows immediately from Lemma 5.6. Relations (5.91) and (5.103) prove Lemma 5.7 for this case. The case $\beta_{1} \geq \beta_{2}$ is similar.

Proof of Theorem 2.1: For the case $\mu_{1}>0$ and $\mu_{2}>0$ this theorem follows directly from 
Lemma 5.7 and Theorem 4.3. The case $\mu_{2}=0$ is the degenerate case, when the service time at the second device vanishes. In this case the virtual service time is given by the simpler formula (2.10) and the construction used above simplifies matters. It is sufficient to use Lemma 5.6 instead of Lemma 5.7. This case was discussed in detail in [9] and we omit the details here. The case $\mu_{1}=0$ of course similar to the case $\mu_{2}=0$.

Note 5.5: Roughly speaking it is possible to say that functions $\Re$ at which the lower bound of the rate function in definition (4.1)-(4.2) of the principle of large deviations is attained describe approximately the structure of fluctuations which largely contributes to the probabilities of large deviations. In our case, they are the functions $\mathfrak{N}$ introduced in the formulation of Lemma 5.7. It is a rigorous interpretation of the discussion in the last paragraph of $\S 2$. However, we do not describe the whole set of functions at which the lower bound in the definition of the principle of large deviations is attained. It seems that, in the general case, this set contains a unique point $\mathfrak{N}$, but in some degenerate cases (for example if $\beta_{1}=\beta_{2}$ ) it can be essentially wider.

\section{References}

[1] Borovkov, A.A., Stochastic Processes in Queueing Theory, Nauka, Moscow 1972, (in Russian). (Engl. trans. Springer-Verlag, New York, Berlin 1976).

[2] Boxma, O.J., On a tandem queueing model with identical service times at both counters, I, Adv. Appl. Prob. 11 (1979), 616-643.

[3] Boxma, O.J., On a tandem queueing model with identical service times at both counters, II, Adv. Appl. Prob. 11 (1979), 644-659.

[4] Burk, P.J., Outprocesses and tandem queues, Proc. Symp. on Computer-Commun. Networks and Teletraffic, Ed. J. Fox, Polytech Inst., Brooklyn, New York 1972, 419-429.

[5] Cramér, H., Sur un nouveau theoreme-limite de la theorie des probabilities, Actualites Scientifiques et Industrielles 763, Colloque consacre a la theorie des probabilities, vol 3, Hermann, Paris 1938, 5-23.

[6] Cramér, H., On some questions connected with mathematical risk, Univ. Calif. Publ. in Statistics, 2 (1954), 99-125.

[7] Dembo, A., Zeitouni, O, Large Deviations Techanique and Applications, Jones and Bartlett Publishers, Boston, London 1992.

[8] Deuschel, J.-D., Stroock, D.W., Large Deviations, Academic Press, New York 1989.

[9] Dobrushin, R.L., Pechersky, E.A., Large deviations for random processes with independent increments on infinite intervals, Proc. of the Kolmogorov Probability and Statistics Semester, Gordon and Breach 1994 (in print).

[10] Friedman, H.D., Reduction methods for tandem queueing systems, Oper. Res. 13 (1965), 121-131.

[11] Gihman, I.I., Skorohod, A.V., The Theory of Stochastic Processes, Vol. II, SpringerVerlag, Berlin 1975.

[12] Klimov, G.P., Some resolved and unresolved problems for service by a consecutive chain of servers, Izv. of AN SSSR, Techn. Kib. 6 (1970), 88-92 (in Russian).

[13] Lipster, R. Sh., Pukhalskii, A.A., Limit theorems on large deviations for semimartingales, Stoch. Stoch. Rep. 38 (1992), 201-249.

[14] Loynes, R.M., The stability of a system of queues in series, Proc. Cambridge Phil. Soc. 60 (1964), 569-574.

[15] Lynch, J., Sethuraman, J., Large deviations for processes with independent increments, Ann. Prob. 15 (1987), 610-627.

[16] Mogulskii, A.A., Large deviations for processes with independent increments, Ann. Prob. 21 (1993), 202-215.

[17] Pukhalskii, A., On functional principle of large deviations, New Trends in Probability and 
Statistics, eds. V. Sazonov and T. Shervashidze, VSP/Mokslas 1991, 198-219.

[18] Rockafellar, R.T., Convex Analysis, Princeton University Press, Princeton, NJ 1970.

[19] Tsoucas, P., Rare events in series of queues, J. Appl. Prob. 29 (1992), 168-175.

[20] Walrand, J., An Introduction to Queueing Networks, Prentice-Hall, Englewood Cliffs, NJ 1988. 


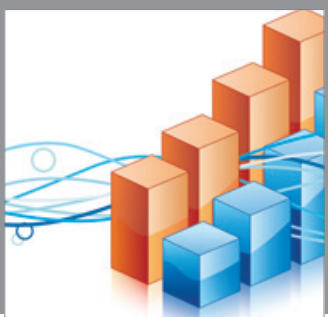

Advances in

Operations Research

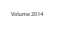

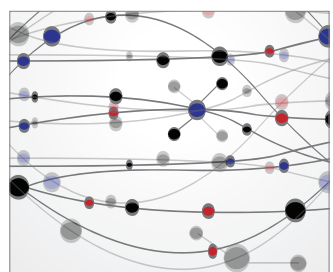

\section{The Scientific} World Journal
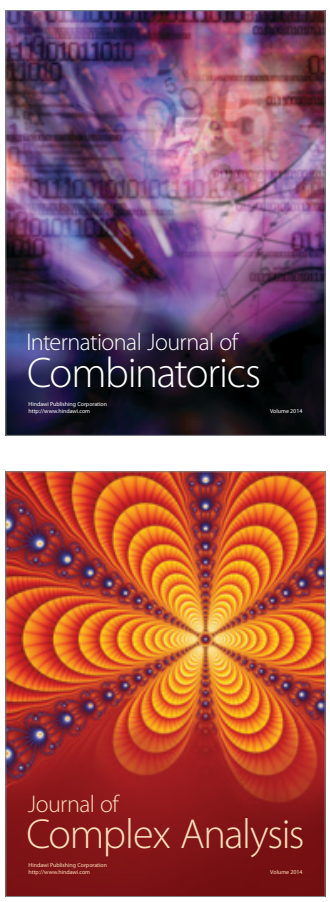

International Journal of

Mathematics and

Mathematical

Sciences
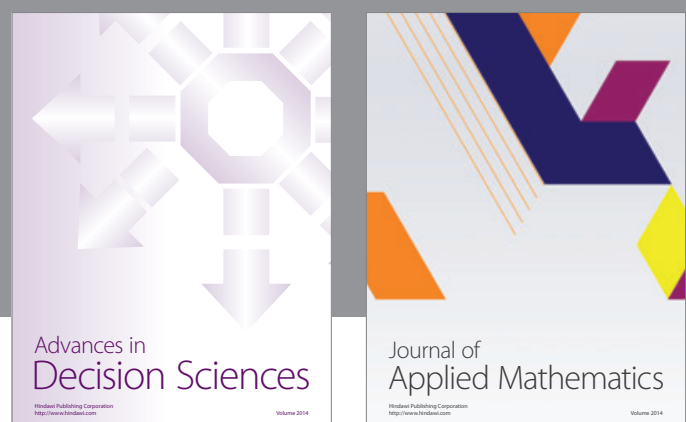

Journal of

Applied Mathematics
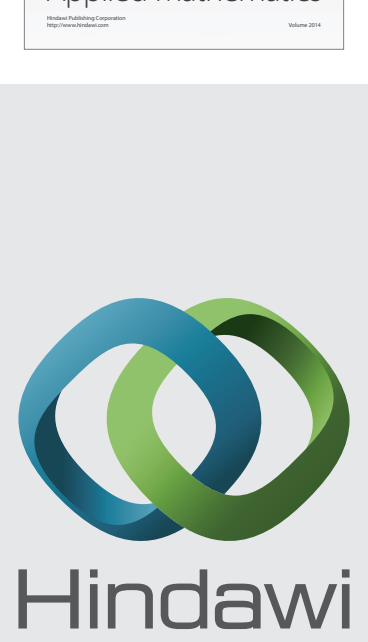

Submit your manuscripts at http://www.hindawi.com
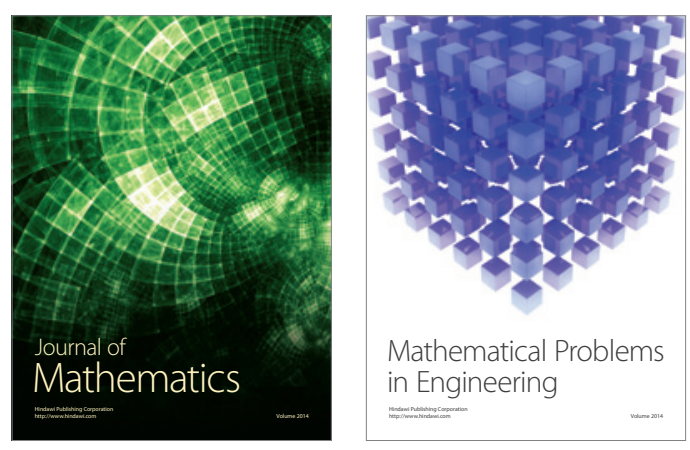

Mathematical Problems in Engineering
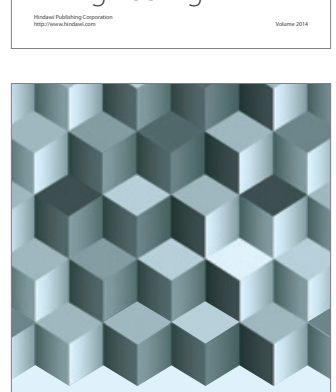

Journal of

Function Spaces
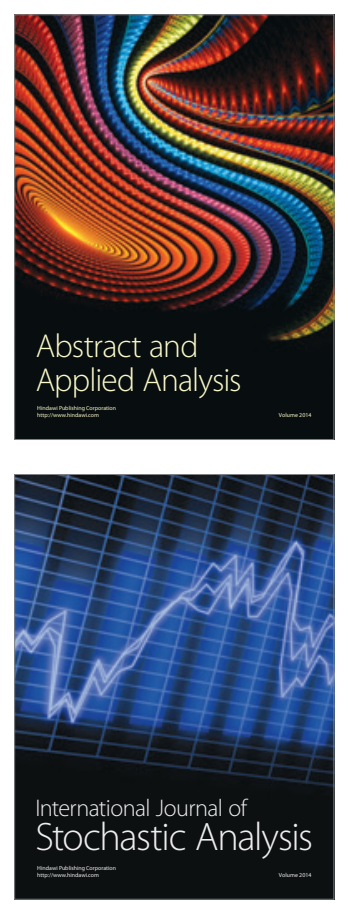

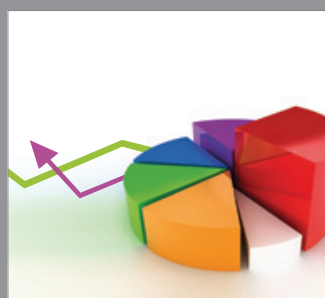

ournal of

Probability and Statistics

Promensencen
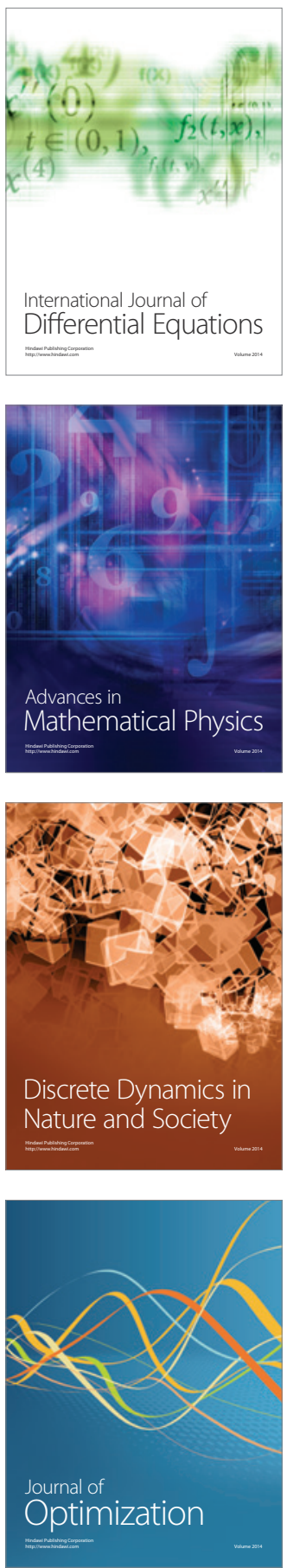